\title{
The Faint End of the Centaurus A Satellite Luminosity Function
}

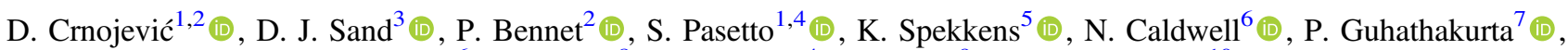

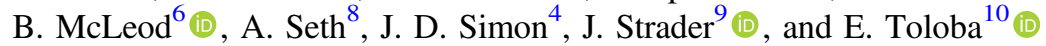 \\ ${ }^{1}$ University of Tampa, 401 West Kennedy Boulevard, Tampa, FL 33606, USA; dcrnojevic@ut.edu \\ ${ }^{2}$ Department of Physics \& Astronomy, Texas Tech University, Box 41051, Lubbock, TX 79409-1051, USA \\ ${ }^{3}$ Department of Astronomy and Steward Observatory, University of Arizona, 933 N. Cherry Avenue, Tucson, AZ 85719, USA \\ ${ }^{4}$ Observatories of the Carnegie Institution for Science, 813 Santa Barbara Street, Pasadena, CA 91101, USA \\ ${ }^{5}$ Department of Physics and Space Science, Royal Military College of Canada, Box 17000, Station Forces, Kingston, ON K7L 7B4, Canada \\ ${ }^{6}$ Harvard-Smithsonian Center for Astrophysics, Cambridge, MA 02138, USA \\ ${ }^{7}$ Department of Astronomy \& Astrophysics, UCO/Lick Observatory, University of California Santa Cruz, 1156 High Street, Santa Cruz, CA 95064, USA \\ ${ }^{8}$ Department of Physics and Astronomy, University of Utah, Salt Lake City, UT 84112, USA \\ ${ }^{9}$ Department of Physics and Astronomy, Michigan State University, East Lansing, MI 48824, USA \\ ${ }^{10}$ Department of Physics, University of the Pacific, 3601 Pacific Avenue, Stockton, CA 95211, USA \\ Received 2018 September 6; revised 2018 December 30; accepted 2019 January 2; published 2019 February 12
}

\begin{abstract}
The Panoramic Imaging Survey of Centaurus and Sculptor (PISCeS) is constructing a wide-field map of the resolved stellar populations in the extended halos of these two nearby, prominent galaxies. We present new Magellan/Megacam imaging of a $\sim 3 \mathrm{deg}^{2}$ area around Centaurus A (Cen A), which filled in much of our coverage to its south, leaving a nearly complete halo map out to a projected radius of $\sim 150 \mathrm{kpc}$ and allowing us to identify two new resolved dwarf galaxies. We have additionally obtained deep Hubble Space Telescope (HST) optical imaging of 11 out of the 13 candidate dwarf galaxies identified around Cen A and presented in Crnojević et al. 2016a: seven are confirmed to be satellites of Cen A, while four are found to be background galaxies. We derive accurate distances, structural parameters, luminosities, and photometric metallicities for the seven candidates confirmed by our $H S T /$ ACS imaging. We further study the stellar population along the $\sim 60 \mathrm{kpc}$ long (in projection) stream associated with Dw3, which likely had an initial brightness of $M_{V} \sim-15$ and shows evidence for a metallicity gradient along its length. Using the total sample of 11 dwarf satellites discovered by the PISCeS survey, as well as 13 brighter previously known satellites of Cen A, we present a revised galaxy luminosity function for the Cen A group down to a limiting magnitude of $M_{V} \sim-8$, which has a slope of $-1.14 \pm 0.17$, comparable to that seen in the Local Group and in other nearby groups of galaxies.
\end{abstract}

Key words: galaxies: dwarf - galaxies: evolution - galaxies: groups: individual (Cen A) - galaxies: halos galaxies: luminosity function, mass function - galaxies: photometry

\section{Introduction}

Observations on large scales ( $\gtrsim 10 \mathrm{Mpc})$ are consistent with a universe dominated by dark energy and cold dark matter (CDM), along with a small baryonic component (e.g., Planck Collaboration et al. 2016). Within this $\Lambda$ CDM model for structure formation, galaxies grow hierarchically within dark matter halos (e.g., Springel et al. 2006), and many detailed galaxy properties are now reproduced in dark matter simulations that include the effects of baryonic physics (e.g., Vogelsberger et al. 2014). However, on scales comparable to and below the size of individual galaxy halos $(\lesssim 1 \mathrm{Mpc})$, significant challenges to the $\Lambda \mathrm{CDM}$ framework have been raised (for a recent review, see Bullock \& Boylan-Kolchin 2017), including the "missing satellites problem" (Klypin et al. 1999; Moore et al. 1999), the "too big to fail" problem (Boylan-Kolchin et al. 2011), and the apparent planes of satellites around nearby galaxies (e.g., Pawlowski et al. 2012; Ibata et al. 2013; Pawlowski \& Kroupa 2013; Müller et al. 2018).

Significant progress has been made in addressing the smallscale challenges to the $\Lambda \mathrm{CDM}$ paradigm on both the theoretical and observational fronts. Numerical simulations that include a sophisticated treatment of baryonic physics show improved comparisons with dwarf galaxies in the Local Group (e.g., Brooks et al. 2013; Sawala et al. 2016; Wetzel \& Hopkins 2016), while a critical assessment of the completeness limit of current searches for dwarf galaxies around the Milky Way (MW) indicates that the "missing satellites problem" is not as severe as initially thought (Koposov et al. 2008; Tollerud et al. 2008; Hargis et al. 2014; Kim et al. 2018). Meanwhile, further Local Group dwarf galaxy discoveries (e.g., most recently Drlica-Wagner et al. 2016; Koposov et al. 2018; Torrealba et al. 2018) add to the current total and point to a rich bounty of new satellites in the era of the Large Synoptic Survey Telescope.

To fully test the $\Lambda \mathrm{CDM}$ paradigm on subgalactic scales, however, we must also look beyond the Local Group to measure dwarf and other substructure properties around primary halos with different masses, morphologies, and environments. Recent progress has been made in several nearby systems using deep, wide-field imaging (e.g., Chiboucas et al. 2009; Crnojević et al. 2014b, 2016a; Sand et al. 2014, 2015b; Carlin et al. 2016; Toloba et al. 2016; Bennet et al. 2017; Carrillo et al. 2017; Danieli et al. 2017; Smercina et al. 2017, 2018), as well as widefield spectroscopy (Geha et al. 2017). Searches for isolated dwarf galaxies provide further constraints on reionization effects and on galaxy formation mechanisms, for instance, in the absence of tidal and ram pressure stripping (Sand et al. 2015a; Tollerud et al. 2015; Janesh et al. 2017; Tollerud \& Peek 2018).

Centaurus A (Cen A) is the closest accessible elliptical galaxy, and it is the central galaxy of a relatively rich group (e.g., Karachentsev et al. 2007). We have thus chosen Cen A as one of the targets of our Panoramic Imaging Survey of Centaurus and Sculptor (PISCeS), a wide-field imaging survey using the 
Megacam imager at the Magellan Clay telescope. One of the principal goals of PISCeS is to identify new, faint dwarf galaxies around Cen A and around the spiral Sculptor (NGC 253; located in a loose group of galaxies) by imaging their resolved stellar populations and compare the properties of these dwarfs to those of Local Group and simulated dwarfs. In previous work around Cen A, we have highlighted a pair of faint satellites at $D \approx 90 \mathrm{kpc}$ in projection (Crnojević et al. 2014b) and have presented a comprehensive red giant branch (RGB) star map of Cen A's halo, highlighting new streams, dwarfs galaxies, and other halo substructures (Crnojević et al. 2016a). Our work represents the most complete census of the halo stellar populations and the satellites within $\sim 150 \mathrm{kpc}$ of Cen A. Here we present two new dwarf galaxy candidates from our 2017 observing season, which focused on extending the spatial coverage of the survey to the south of Cen A. We also present Hubble Space Telescope (HST) follow-up of 11 dwarf galaxy candidates, along with a detailed look at a disrupting dwarf galaxy and its associated stellar stream. HST follow-up of our ground-based discoveries allows for improved distance, structural parameters, and luminosity measurements and, in some cases, is necessary for determining whether the PISCeS groundbased candidates are indeed dwarf galaxies at the distance of Cen A. Based on the results from these HST data and our continuing ground-based campaign, we provide a preliminary estimate of the dwarf galaxy luminosity function (LF) of Cen A to compare with those calculated for other Local Volume groups of galaxies. Throughout this work, we assume a Cen A distance of $D=3.8 \mathrm{Mpc}$ (Harris 2010).

\section{The PISCeS Survey}

We begin by briefly describing the PISCeS survey as it pertains to Cen A. For more details on our survey strategy and observational methods, see Crnojević et al. (2014b, 2016a), and for our preliminary results around the nearby spiral galaxy NGC 253, see Sand et al. (2014) and Toloba et al. (2016).

The ultimate observational goal of PISCeS is to image the halos of Cen A and NGC 253 in the Sculptor group out to a projected radius of $D \sim 150 \mathrm{kpc}$, deep enough to resolve $\sim 1-2$ mag below the tip of the RGB (TRGB) (limiting magnitudes: $g$, $r \approx 26-26.5 \mathrm{mag})$. The data of this areal coverage are comparable to those of the Pan-Andromeda Archaeological Survey of M31 (e.g., McConnachie et al. 2009), although 3 mag less deep in absolute magnitude. This allows for direct comparison of the satellite and substructure properties between the Cen A and M31 systems and extends our general knowledge of substructure to new systems and environments.

To image the outer halo of Cen A, we mosaic individual pointings of the Megacam imager (McLeod et al. 2015) on the Magellan Clay telescope. Megacam has a $\sim 24^{\prime} \times 24^{\prime}$ field of view (FOV), and a typical pointing is observed for $6 \times 300 \mathrm{~s}$ in each of the $g$ and $r$ bands. The data are reduced in a standard way by the Smithsonian Astrophysical Observatory Telescope Data Center (see McLeod et al. 2015; Crnojević et al. 2016a, for further details), and point-spread function (PSF) photometry is performed on the stacked final images using the DAOPHOT and ALLFRAME software suite (Stetson 1987, 1994). Instrumental magnitudes are calibrated to the Sloan Digital Sky Survey system using standard star field observations obtained on photometric nights; calibration of the overall survey is facilitated by small pointing overlaps between adjacent fields

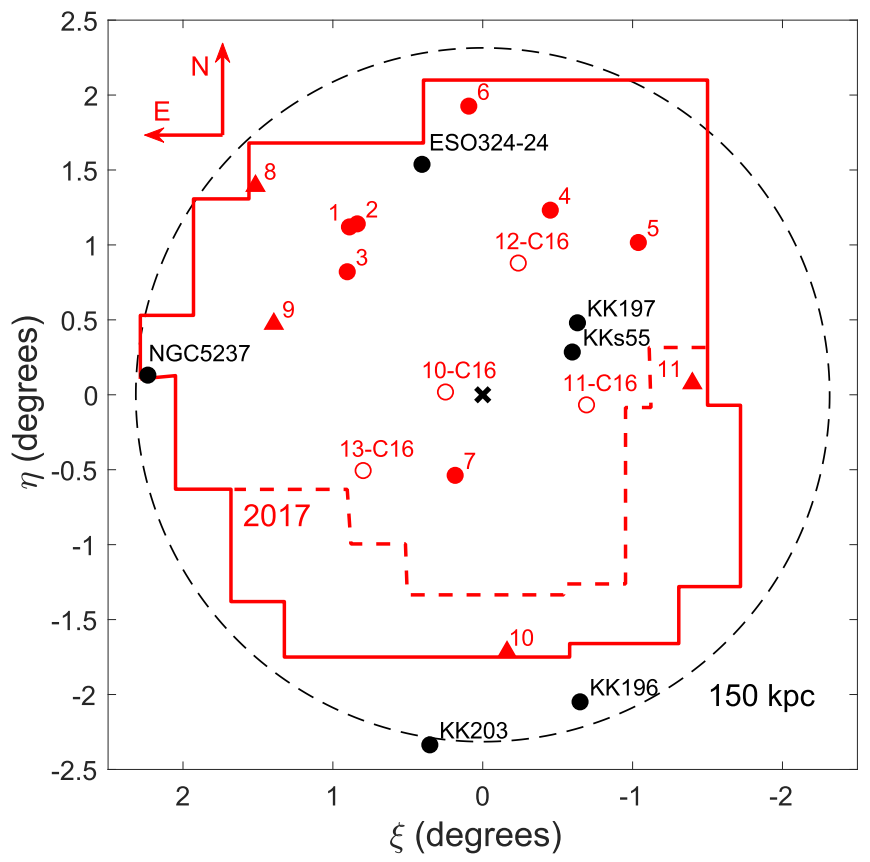

Figure 1. Footprint of the PISCeS survey to date (red polygon) in standard coordinates centered on Cen A (black cross); the area surveyed in our latest observing run (2017; i.e., the area not reported in Crnojević et al. 2016a) is to the south of the red dashed line; the $150 \mathrm{kpc}$ projected galactocentric radius is shown as a black dashed circle. Cen A dwarfs that were known prior to the PISCeS survey are plotted as filled black circles; confirmed satellites discovered in PISCeS are plotted as filled red circles or triangles (numbered following their names, e.g., 1 is for CenA-MM-Dw1), where the triangles indicate dwarfs that have not been observed with $H S T$; open red circles are candidate satellites from Crnojević et al. (2016a) that turned out to be background objects as deduced from HST imaging (see Section 2 for the nomenclature convention).

(typically $\sim 2$ !). Artificial star tests are run on all images to quantify completeness and magnitude uncertainties.

The status of the Cen A PISCeS program as of 2017 can be seen in Figure 1. The data obtained prior to 2017 were published in Crnojević et al. (2016a), where 13 new dwarf candidates were presented (along with other halo substructures). These dwarf candidates span an absolute magnitude range of $-7.2>M_{V}>-13.0$ and half-light radius range of 220-2900 pc, shedding light on Cen A's faint satellite population $\left(M_{V} \gtrsim-10\right)$ for the first time. Here we present $H S T$ follow-up imaging of 11 of these dwarf galaxy candidates to confirm their status as Cen A dwarfs and to precisely measure their physical properties. We also present $H S T$ pointings along the dramatic tidal stream associated with Dw3. In the next section, we present further results of our 2017 ground-based Megacam campaign, focused on filling in our southern coverage of Cen A's halo, where we discovered two new dwarf galaxies that are discussed here for the first time.

We have obtained data in 2018 to complete our survey out to a galactocentric radius of $\sim 150 \mathrm{kpc}$. The data reduction for that data set is still in progress and will be presented elsewhere; however, a visual inspection of the images does not reveal any new candidate dwarf satellite in the remaining surveyed area.

\section{The Magellan 2017 Data Set: Two New Cen A Dwarfs}

We continued the PISCeS campaign around Cen A in 2017, observing with Megacam/Magellan Clay on the nights of 2017 April 20-24 (UT). During the span of these five nights, we collected data for 20 new fields, focused on filling in areas 
south of Cen A, as can be seen by the dashed region in Figure 1 (which shows a total of 95 pointings obtained up to the 2017 campaign). The data for the 20 new Megacam fields were generally obtained in photometric conditions, with the seeing ranging from 0.5 to $0.9 \operatorname{arcsec}(g$ band). The data were reduced as described in Crnojević et al. (2016a) and Section 2.

We found two new dwarf galaxy candidates in the 2017 Magellan data set, which are marked in Figure 1. Since some of our dwarf candidates from Crnojević et al. (2016a) turned out to be background objects (see Section 4.2), we rename those Dw10-C16 to Dw13-C16 (from the original Crnojević et al. 2016a nomenclature), and we dub the two new discoveries CenA-MM17-Dw10 and CenA-MM17-Dw11, or to follow the original nomenclature, Dw10 and Dw11. These dwarfs were first found via visual inspection and then confirmed based on the RGB map of stars consistent with the distance to Cen A in each field, where they stand out as clear overdensities. We show the dereddened color-magnitude diagram (CMD) and RGB spatial map of each dwarf in Figure 2. Both objects are faint but clearly detected above the background. Each consists of an old stellar population (the isochrones shown in Figure 2 are of a $12 \mathrm{Gyr}$ old, $[\mathrm{Fe} / \mathrm{H}]=-1.5$ stellar population; Dotter et al. 2008) with no signs of recent star formation.

We measure the distance to Dw10 and Dw11 as described in Crnojević et al. (2016a). We use the standard TRGB method (e.g., Da Costa \& Armandroff 1990; Lee et al. 1993; see also further discussion in Section 4.3 in relation to our HST data), measuring a discontinuity in its LF with a Sobel edge-detection filter. The newly discovered dwarfs, Dw10 and Dw11, have TRGB distances consistent with Cen A (Table 1), confirming their association. In projection, Dw10 and Dw11 are 110 and $\sim 90 \mathrm{kpc}$ (or 1.72 and $1.40 \mathrm{deg}$ ) from Cen A, respectively.

To measure the structural parameters and luminosities of Dw10 and Dw11, we use the method of moments as presented in Crnojević et al. (2014a). First, the surface brightness profile of each dwarf is found by summing the area-normalized flux of stars within an RGB selection box as a function of radius, correcting for field contamination and incompleteness based on our artificial star tests. In order to correct for unresolved light from stars below our detection limit, we directly measure the image flux within a central aperture and rescale the surface brightness profile to match it. This rescaled surface brightness profile is fit to an exponential using least-squares minimization in order to measure the half-light radius and central surface brightness. Finally, the absolute magnitude is computed by integrating the best-fit exponential profile. The final derived quantities for Dw10 and Dw11 are presented in Table 1. The properties of these two new dwarfs are broadly consistent with the dwarf population found in our earlier work (Crnojević et al. 2016a).

We also searched the H I Parkes All Sky Survey (HIPASS; Barnes et al. 2001) for neutral gas at the position of the two new dwarfs, and we present their $5 \sigma$ upper limits in Table 1 . As with the H I limits of the other Cen A dwarfs found by PISCeS (Crnojević et al. 2016a), the HIPASS data are not sensitive enough to confirm or exclude the presence of $\mathrm{HI}$ in these dwarfs below $3 \times 10^{6} M_{\odot}$.

\section{HST Imaging: Data and Photometry}

Including the discovery of Dw10 and Dw11, PISCeS has uncovered 15 dwarf candidates around Cen A (see Table 2). As mentioned above, we obtained HST observations of 11 of the
13 dwarf candidates found in Crnojević et al. (2016a), which are marked as red circles (both filled and open) in Figure 1.

HST follow-up imaging was obtained with the Wide Field Channel (WFC) of the Advanced Camera for Surveys (ACS). Most of the targets were observed as part of the program GO13856 (PI: Crnojević), with the exception of CenA-MM-Dw3, which was observed as part of program GO-14259 (PI: Crnojević); see Table 2 for a summary. Each target was observed for a total of one orbit (two orbits for CenA-MMDw3) in the F606W and F814W filters, which broadly correspond to the Johnson-Cousins $V$ and $I$ bands (exposure times of $\sim 1100$ and $2500 \mathrm{~s}$ per filter, for one and two orbits, respectively).

Parallel observations were simultaneously obtained with the Wide Field Camera 3 (WFC3) UVIS channel, with the same filters and similar exposure times. The parallel pointings serve as background/foreground fields (to clean the CMDs from contaminating resolved objects), as well as control fields of the Cen A halo (to study the halo properties, including possible stellar population gradients).

\subsection{Photometry}

PSF photometry was performed on the pipeline-produced.$f t$ images with the latest version (2.0) of the dedicated photometric package DOLPHOT (Dolphin 2002). Generally, we adopt the input parameters suggested by the DOLPHOT User's Guide for each camera, including the corrections for charge transfer efficiency losses, which are substantial for both ACS and WFC3. For the most crowded of our pointings (the one targeting Dw7, i.e., the candidate satellite closest to the center of Cen A in projection, at $\sim 0.5 \mathrm{deg}$ ), we set the parameters FitSky $=3$ and img_RAper $=10$ to improve the sky-fitting procedure. The photometry is then culled with the following criteria: the sum of the crowding parameters in the two bands is $<1$ (or $<0.6$ for the crowded photometry case), the squared sum of the sharpness parameters in the two bands is $<0.075$, and the photometric errors as derived by DOLPHOT are $\lesssim 0.3$ in each band.

We subsequently perform artificial star tests in order to accurately assess photometric errors and incompleteness in the $H S T$ data. The artificial stars are distributed evenly both spatially and in color-magnitude space and extend as faint as 2 mag below the faintest detected stars (after quality cuts) to account for objects upscattered into the detectable magnitude range as a result of blending and noise. For each field, we inject a number of artificial stars between a minimum of 200,000, in order to ensure a robust statistics, and a maximum of 1,200,000, i.e., 10 times the number of sources (after quality cuts) in the most crowded of our pointings (note that DOLPHOT adds one fake star at a time in order not to increase crowding artificially). Photometry and quality cuts are performed in the same exact way as done for the original photometry; photometric errors are shown for each galaxy in the CMDs of the next section. Finally, representative completeness curves are shown in Figure 3 (the maximum completeness value is below $100 \%$ because of spatial incompleteness).

\subsection{Satellite Confirmation and Contaminants from HST Imaging}

Seven Cen A satellites were resolved into stars in our Magellan imaging (Crnojević et al. 2014b, 2016a) and have now been followed-up with our HST program and confirmed as 

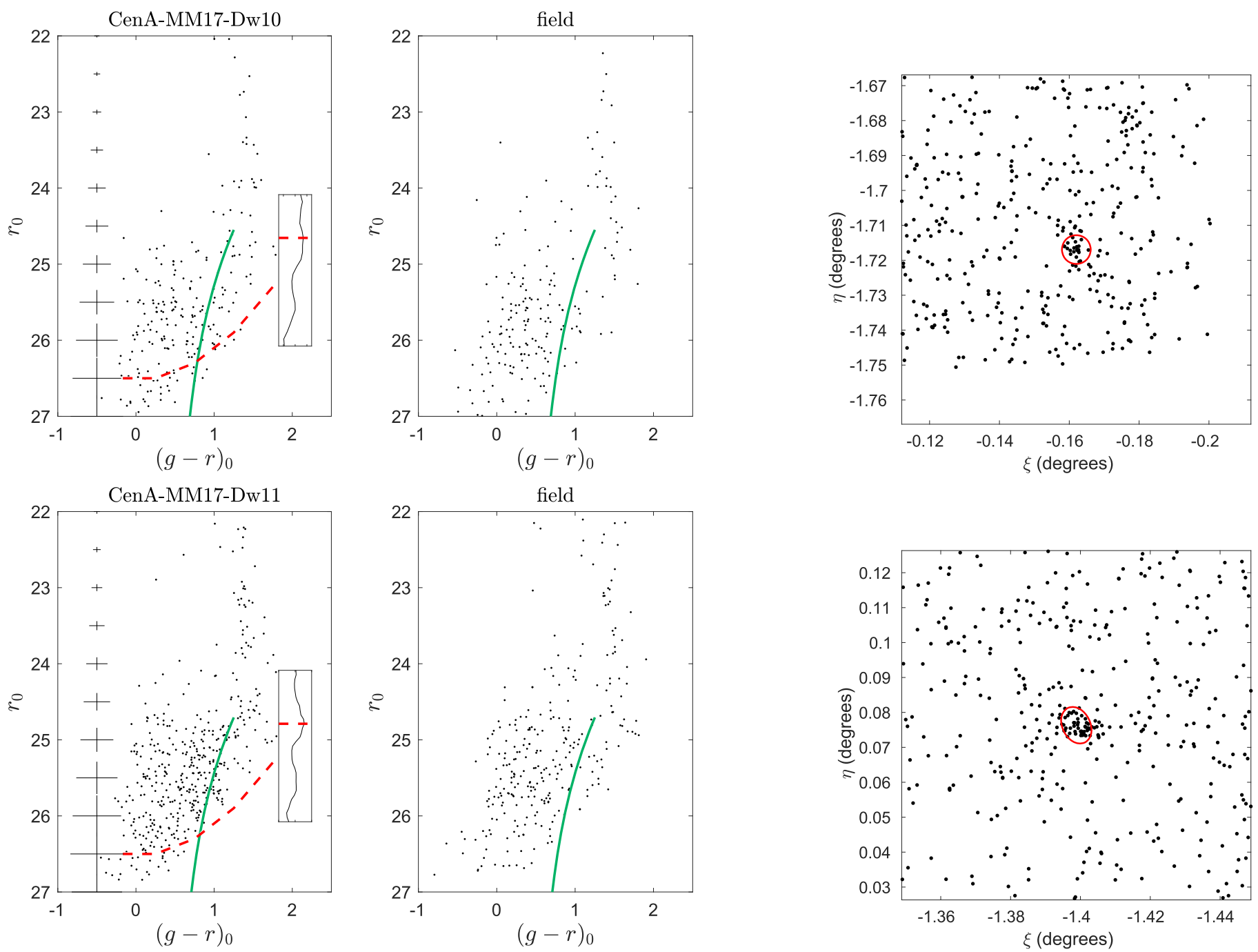

Figure 2. Dereddened Magellan/Megacam CMDs for CenA-MM17-Dw10 (top panels) and CenA-MM17-Dw11 (bottom panels). We plot stars within a box of $0 ! 6 \times 00^{\prime} 6$ and $0 ! 9 \times 0 ! 9$ centered on Dw10 and Dw11, respectively. Isochrones with a $12 \mathrm{Gyr}$ age and a metallicity $[\mathrm{Fe} / \mathrm{H}]=-1.5(\mathrm{Dotter}$ et al. 2008$)$ are shifted to the measured distance of each dwarf. The red dashed line indicates the 50\% completeness level, and photometric errors as derived from artificial star tests are shown on the left side of the CMD. The inset plots in the left panels show the LF after convolution with a Sobel filter, as well as the derived TRGB magnitude (red dashed line). A background field CMD drawn from the Magellan pointing containing the dwarf and rescaled in area is shown for comparison in the middle panels. In the right panels, we show a $3 \times 3^{\prime 2}$ cutout of the dwarf RGB stars' spatial distribution in the Magellan/Megacam pointings, centered on the dwarfs. The red circles indicate each dwarf's measured half-light radius.

group members. The deep CMDs obtained with the ACS camera reveal stellar populations consistent with those expected at the group's distance (see Figure 4), and we show the individual dereddened CMDs for dwarfs Dw1 to Dw7 in Figures 5-10.

In Crnojević et al. (2016a), we reported on four candidate dwarfs detected as surface brightness enhancements without a resolved stellar counterpart (Dw10-Dw13). Our follow-up HST imaging confirmed the lack of resolved populations in these targets, thus excluding the possibility that they are low-mass satellites of Cen A and are instead mostly background galaxies. In the case of Dw13, examination of the HST images (in particular, the lack of a structured surface brightness enhancement) has led us to conclude that the object is galactic cirrus and not a genuine background object (e.g., Guhathakurta \& Tyson 1989). We rename these contaminant objects Dw10-C16 to Dw13-C16 (from the original Crnojević et al. 2016a nomenclature) to avoid confusion with the new candidate satellites discovered in our ongoing survey, which are now dubbed Dw10, Dw11, and so on (see Section 3). Because of the very low surface brightness of these background objects, integrated photometry from the HST images is very challenging, and we thus use the Magellan/Megacam images to derive their luminosities and structural properties. We model them with GALFIT (Peng et al. 2002), following the procedure adopted in Bennet et al. (2017), and the derived values are reported in Table 3. Among these unresolved objects, Dw11C16 is noteworthy: given its low surface brightness and large effective radius, this galaxy would be classified as an ultradiffuse galaxy if located at distances $\gtrsim 26 \mathrm{Mpc}$ (distances cannot be constrained for unresolved objects with our data set); Dw10-C16 would also qualify as an ultradiffuse galaxy, but only at larger distances $\gtrsim 80 \mathrm{Mpc}$. Finally, we have searched a region around each of these objects in NED to investigate a possible association with known galaxies, but we have found them to be isolated.

Given that all unresolved candidate dwarfs identified in PISCeS turned out to be background objects, we conclude that 
Table 1

Properties of New PISCeS 2017 Dwarfs

\begin{tabular}{lcc}
\hline \hline Parameter & Dw10 & Dw11 \\
\hline R.A. (h:m:s) & $13: 24: 32^{\prime \prime} 9 \pm 1^{\prime \prime}$ & $13: 17: 49{ }^{\prime \prime} .2 \pm 1^{\prime \prime}$ \\
Decl. (d:m:s) & $-44: 44: 07 ! .1 \pm 2^{\prime \prime}$ & $-42: 55: 36 ! .8 \pm 8^{\prime \prime}$ \\
$E_{(B-V)}$ & 0.09 & 0.12 \\
$(m-M)_{0}(\mathrm{mag})$ & $27.57 \pm 0.29$ & $27.73 \pm 0.22$ \\
$D(\mathrm{Mpc})$ & $3.27_{-0.46}^{+0.41}$ & $3.52_{-0.37}^{+0.33}$ \\
$D_{\mathrm{CenA}, \mathrm{proj}}(\mathrm{deg})$ & 1.72 & 1.40 \\
$D_{\mathrm{CenA}, \mathrm{proj}}(\mathrm{kpc})$ & 112 & 91 \\
$\epsilon$ & $<0.27^{\mathrm{a}}$ & $0.27 \pm 0.21$ \\
$r_{h}(!)$ & $0.25 \pm 0.06$ & $0.33 \pm 0.04$ \\
$r_{h}(\mathrm{kpc})$ & $0.24 \pm 0.06$ & $0.34 \pm 0.04$ \\
$\mu_{V, 0}\left(\mathrm{mag} \operatorname{arcsec}{ }^{-2}\right)$ & $26.6 \pm 0.9$ & $25.8 \pm 0.4$ \\
$M_{V}(\mathrm{mag})$ & $-7.8 \pm 1.2$ & $-9.4 \pm 0.6$ \\
$L_{*}\left(10^{5} L_{\odot}\right)$ & $1.1 \pm 1.4$ & $4.7 \pm 2.7$ \\
$M_{\mathrm{H} \mathrm{I}}\left(10^{6} M_{\odot}\right)$ & $\lesssim 4.0$ & $\lesssim 3.1$ \\
$M_{\mathrm{H} \mathrm{I}} / L_{*}\left(M_{\odot} / L_{\odot}\right)$ & $\lesssim 36.7$ & $\lesssim 6.6$ \\
\hline
\end{tabular}

Notes.

a Only an upper limit on the ellipticity, $\epsilon$, was measurable.

b $5 \sigma$ upper limits from HIPASS.

it is unlikely that unresolved candidates in our ground-based imaging are real satellites; this also implies that we expect galaxies with $M_{V} \lesssim-8.0$ (our faintest detected satellite) to be resolved into stars in our survey.

\subsection{Distances from HST Imaging}

The TRGB distance measurement method is widely used for nearby galaxies resolved into stars (e.g., Lee et al. 1993; Sakai et al. 1997; Makarov et al. 2006; Rizzi et al. 2007). It relies on the fact that the I-band LF of old and metal-poor RGB stars presents a sharp break at its bright end that is a robust standard candle, insensitive to metallicity. The absolute magnitude calibration for the TRGB in the HST filter system has been recently revised by Jang \& Lee (2017). Their new value is a factor of two more accurate than previous estimates (e.g., Rizzi et al. 2007). Jang \& Lee (2017) also determined the TRGB color dependence based on deep HST images of eight nearby galaxies:

$$
\begin{aligned}
& M_{\mathrm{F} 814 \mathrm{~W}}^{\mathrm{TRGB}}=-4.015( \pm 0.056)-0.159( \pm 0.01) \\
& \quad \times\left[(F 606 W-\mathrm{F} 814 \mathrm{~W})_{0}-1.1\right]^{2}+0.047( \pm 0.02) \\
& \quad \times\left[(F 606 W-\mathrm{F} 814 \mathrm{~W})_{0}-1.1\right] .
\end{aligned}
$$

We apply this color correction term to our photometry in order to obtain a sharper and more easily measured TRGB (see, e.g., Madore et al. 2009; McQuinn et al. 2016), and we apply our TRGB detection algorithm to this corrected photometry. When deriving TRGB values, we consider stars within one to two half-light radii (the latter case is for dwarfs containing small numbers of stars), and we only consider stars with colors $0.7<(\mathrm{F} 606 \mathrm{~W}-\mathrm{F} 814 \mathrm{~W})_{0}<1.5$. To find the TRGB value, we adopt the approach by Makarov et al. (2006), where a predefined LF is compared to the observed RGB LF. The model LF has the form of two distinct power laws,

$$
\psi=\left\{\begin{array}{ll}
10^{a\left(m-m_{\mathrm{TRGB}}\right)+b}, & m-m_{\mathrm{TRGB}} \geqslant 0, \\
10^{c\left(m-m_{\mathrm{TRGB}}\right)}, & m-m_{\mathrm{TRGB}}<0
\end{array},\right.
$$

where $a$ and $c$ are the slopes of the RGB and asymptotic giant branch (AGB), respectively, and $b$ represents the discontinuity at the TRGB magnitude. The photometric uncertainty, bias, and incompleteness function derived from the artificial star tests are modeled with continuous functions and convolved with the pre-defined LF. We fit the pre-defined function with a nonlinear least-squares method, using a Levenberg-Marquardt algorithm. We use an initial guess for $a, b$ of 0.3 , while for $m_{\mathrm{TRGB}}$ we compute a first estimate with the Sobel filter edge-detection technique described in Sakai et al. (1997). Briefly, the observed LF is binned and smoothed with a Gaussian function following the photometric errors and then convolved with a Sobel filter that highlights the position of the LF edge. This method depends on the chosen binning of the LF, and we thus refine our measurement by fitting the model LF to our data. The latter method gives minimal differences for a range of bin sizes of the observed LF and returns smaller uncertainties on the measured TRGB values.

The TRGB values, the distance moduli, and the distances for our targets are reported in Table 4. The distances derived from the HST data set are slightly higher on average than those derived from the discovery Magellan data set, but mostly consistent within the error bars. The exceptions are Dw7, which is $\sim 0.5$ mag more distant, likely because in the ground-based data set we could not easily separate Cen A's strong contamination from the dwarf's population (this is the closest dwarf in projection to Cen A), and Dw3, which is $\sim 0.4$ mag closer likely because the photometric confusion in the Magellan data set led us to misidentify the brightest RGB stars as luminous AGB stars (the HST CMD shows a less prominent intermediate-age AGB population). The accuracy of the updated $H S T$ distance values is improved by a factor of 2-3 with respect to the Magellan values reported in Crnojević et al. (2016a).

\subsection{Structural Parameters and Luminosities}

We derive the structural parameters for our confirmed Cen A satellites with the maximum likelihood method presented in Martin et al. (2008), using the implementation in Sand et al. (2012). The code fits the selected RGB stars from the HST data (see Figure 4) with an exponential profile with the following free parameters: central coordinates, position angle, ellipticity, half-light radius, and background surface density. The exponential profile is a good fit for all of the confirmed dwarfs. We report the resulting parameters in Table 4; the uncertainties come from boostrap resampling of the data.

In two cases the adopted procedure for the derivation of structural parameters deviates from that just described. For Dw1, the HST data cannot be used to constrain structural parameters, since this dwarf extends well beyond the ACS FOV, and thus the original Magellan photometry is adopted instead. Moreover, Dw1 hosts a nuclear star cluster, which provides an additional cuspy component to its surface brightness profile (A. Seth et al. 2019 , in preparation). For our structural parameter derivation, a small region around the central cluster is thus masked (even though it is not resolved in the ground-based images). For Dw3, which similarly extends well beyond the ACS FOV and is the central region of a $\sim 60 \mathrm{kpc}$ long disrupting dwarf and tidal stream system, we decided to keep the half-light radius from the Magellan photometry (see Crnojević et al. 2016a), which was derived from a fit to the remnant galaxy core, i.e., excluding the tidal tail regions. The central coordinates were derived from the deeper HST photometry with an iterative process, computing 
Table 2

Cen A Satellites Discovered in PISCeS

\begin{tabular}{|c|c|c|c|c|}
\hline Galaxy & Alternative Name & HST Program ID & Confirmed & References \\
\hline Dw1 & CenA-Dw-133013-415321 & 13856 & $\mathrm{y}$ & 1 \\
\hline Dw2 & CenA-Dw-132956-415220 & 13856 & $\mathrm{y}$ & 1 \\
\hline Dw3 & $\cdots$ & 14259 & $\mathrm{y}$ & 2 \\
\hline Dw5 & CenA-Dw-131952-415938 & 13856 & $\mathrm{y}$ & 2 \\
\hline Dw6 & CenA-Dw-132557-410538 & 13856 & $\mathrm{y}$ & 2 \\
\hline Dw7 & CenA-Dw-132628-433318 & 13856 & $\mathrm{y}$ & 2 \\
\hline Dw10-C16 & CenA-Dw-132649-430000 & 13856 & $\mathrm{n}$ & 2 \\
\hline Dw11-C16 & CenA-Dw-132140-430457 & 13856 & $\mathrm{n}$ & 2 \\
\hline Dw12-C16 & CenA-Dw-132410-420823 & 13856 & $\mathrm{n}$ & 2 \\
\hline Dw13-C16 & CenA-Dw-132951-433109 & 13856 & $\mathrm{n}$ & 2 \\
\hline Dw10 & CenA-MM17-Dw10 & $\ldots$ & $\mathrm{y}$ & 3 \\
\hline Dw11 & CenA-MM17-Dw11 & $\ldots$ & $\mathrm{y}$ & 3 \\
\hline
\end{tabular}

References. (1) Crnojević et al. 2014b; (2) Crnojević et al. 2016a; (3) this work.

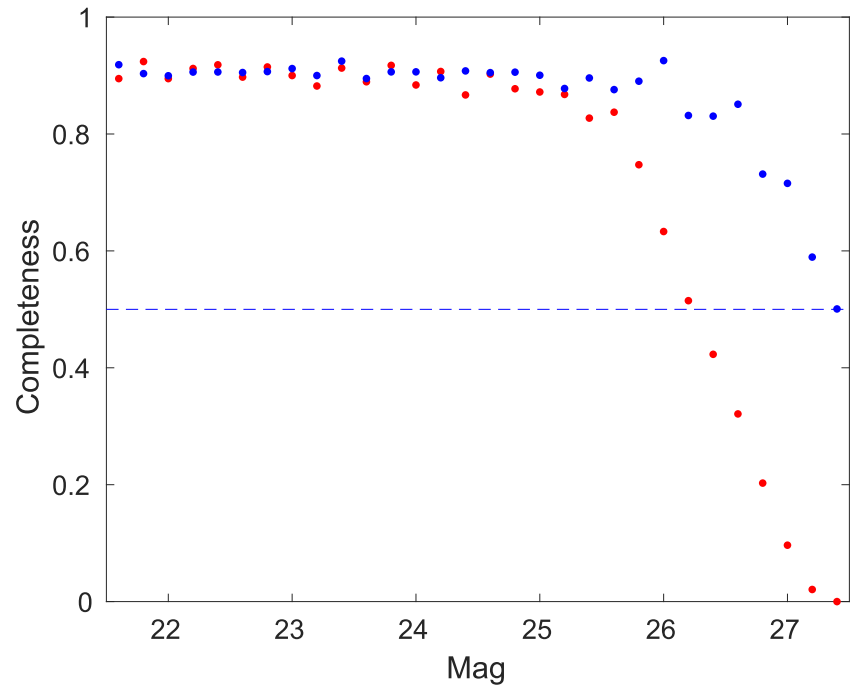

Figure 3. Photometric completeness curves for Dw1 in the F814W (red) and F606W (blue) filters, as determined from our artificial star tests; these are representative for the overall sample of targets.

the average of the stellar positions within circles of decreasing radius, while ignoring the sources found within a radius of 0.15 ! from the central cluster center. We discuss this disrupting dwarf further in Section 5.4.

The absolute magnitude for the confirmed dwarfs is derived as follows. We produce a well-populated $\left(5 \times 10^{6}-\right.$ $5 \times 10^{8} M_{\odot}$, depending on the dwarf) fake population in the HST filters by interpolating Padova isochrones with an old age (10 Gyr) and with the median metallicity derived below for each of the galaxies (see Section 4.5), assuming a Kroupa initial mass function (Kroupa 2001). The fake populations are convolved with photometric errors as derived from the artificial star tests. We then extract stars randomly from this fake population by rescaling the number of stars in the RGB selection box to the observed number of RGB stars within the half-light radius (after subtracting field contaminants). The flux of the extracted fake stars is summed up along the entire LF, in order to account for the faint, unresolved component of the galaxy, and the total luminosity is obtained by multiplying this

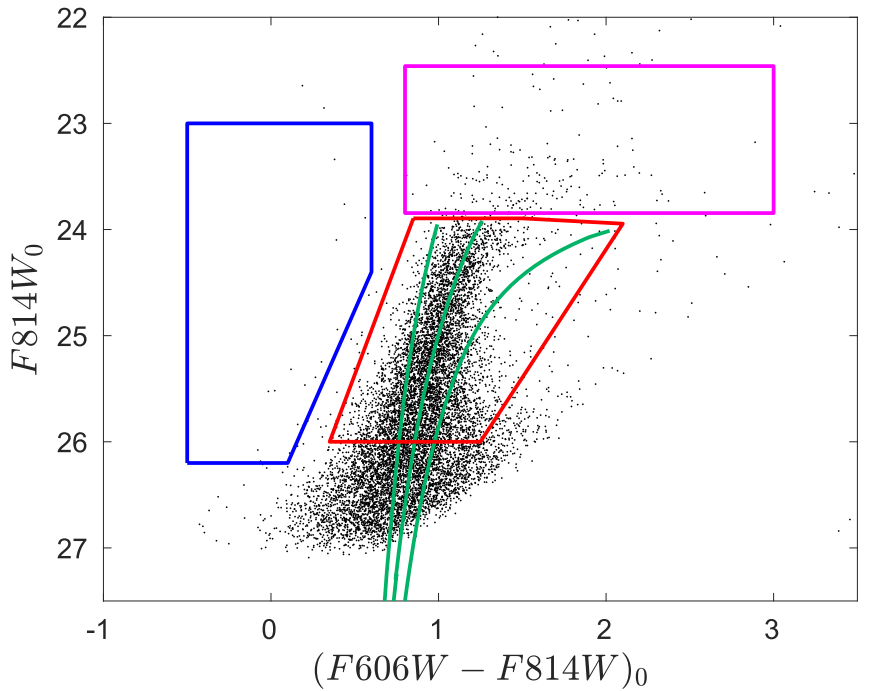

Figure 4. CMD of Dw1, identifying different stellar populations within the target galaxies: old RGB stars (red box; $10 \mathrm{Gyr}$ isochrones with metallicities of $[\mathrm{Fe} / \mathrm{H}]=-2.0,-1.0$, and -0.5 , from left to right), young massive stars $(\lesssim 500 \mathrm{Myr}$, blue box), and intermediate-age upper AGB stars (magenta box). This CMD and the selection boxes are representative for our whole sample of targets.

quantity by 2 . This process is repeated $500 / 1000$ times in order to assess uncertainties; the absolute magnitudes, derived using our computed distance moduli, are converted to a Vegamag $V$-band value by following the Sirianni et al. (2005) prescriptions. We further calculate the central surface brightness values starting from the derived absolute magnitude and the half-light radius, assuming an exponential profile. The final values can be found in Table 4, and Figure 11 shows the relation between the derived absolute magnitudes, half-light radii, and central surface brightnesses as compared to Local Group dwarfs and other galaxy samples.

Overall, the structural parameters and the luminosities derived from the HST data set agree well with the results from the ground-based Magellan imaging to within the uncertainties. For Dw3, Dw4, and Dw6, the absolute magnitudes agree to $\sim 0.1 \mathrm{mag}$. For Dw5 and Dw7, the $H S T$ values are about $1 \mathrm{mag}$ 

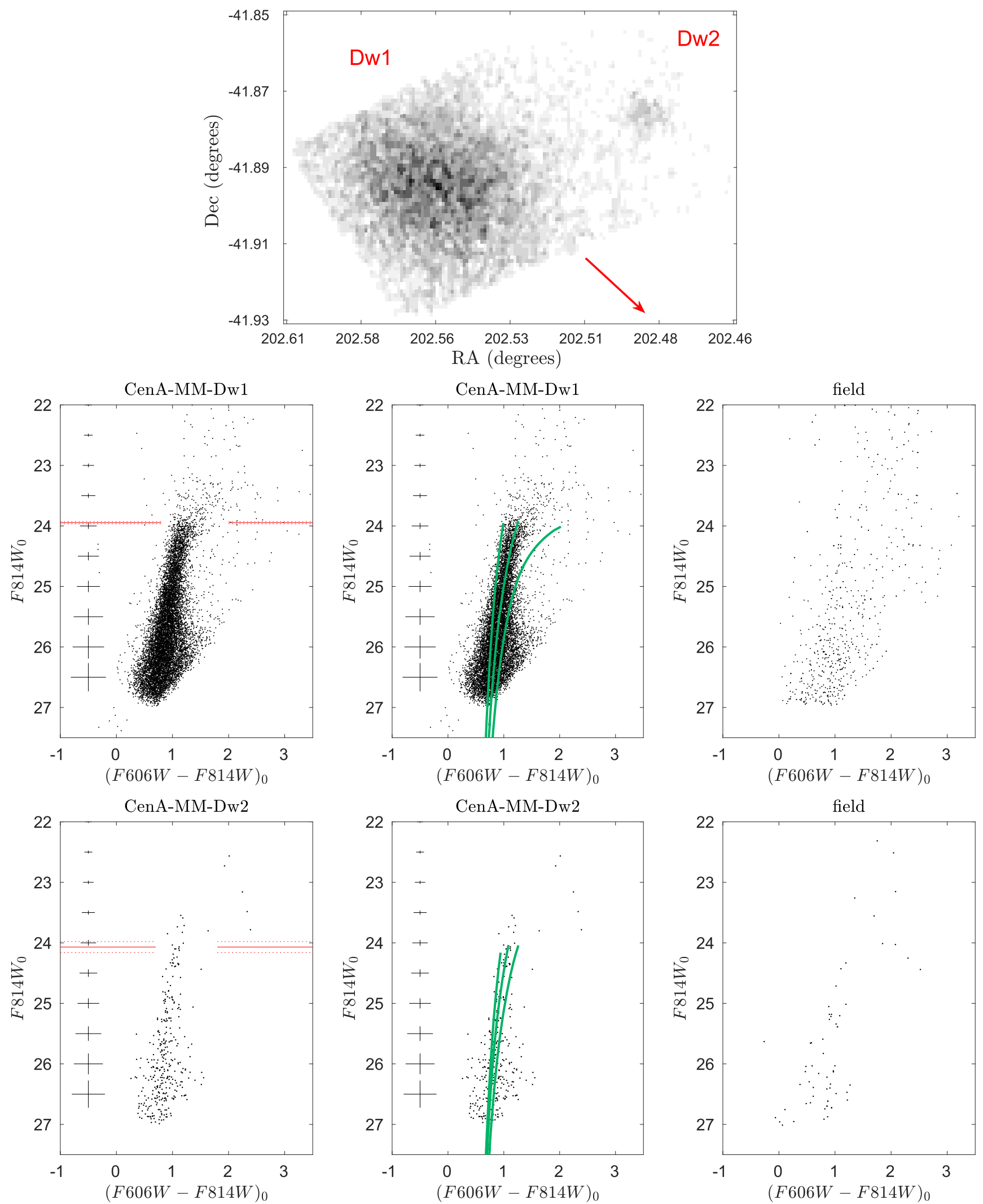

Figure 5. Top panel: stellar density map of RGB stars for the two ACS pointings targeting Dw1 and Dw2 (as labeled), derived for stars within the red selection box in Figure 4. The direction toward Cen A is indicated by the red arrow. The former clearly overfills the ACS FOV. Middle and bottom panels: CMDs of Dw1 and Dw2 including stellar sources within $0.5 r_{h}$ and $r_{h}$, respectively; we also report photometric errors as derived from artificial star tests. In the left panels, we draw the TRGB magnitude and the relative uncertainties (red lines and red dotted lines); in the middle panels, we overplot $10 \mathrm{Gyr}$ isochrones with metallicities of $[\mathrm{Fe} / \mathrm{H}]=-2.0$, -1.0 , and -0.5 for Dw1 and $-2.5,-1.5$, and -1.0 for Dw2 (green lines; Dotter et al. 2008); in the right panels, an area-scaled field CMD is shown. 

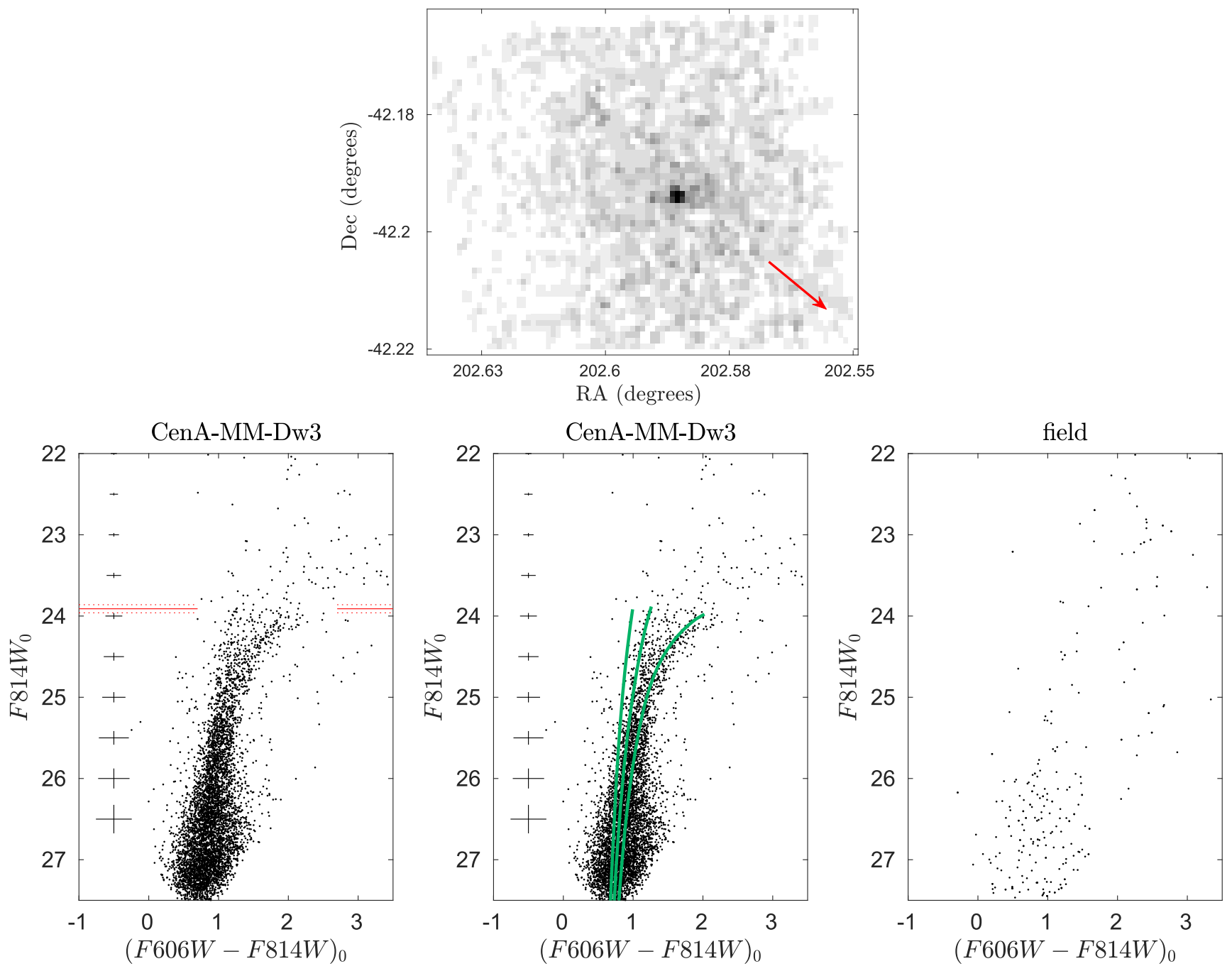

Figure 6. Same as Figure 5, but for the heavily disrupting satellite Dw3. The CMD contains populations within $0.5 r_{h}$, and the isochrones are for $[\mathrm{Fe} / \mathrm{H}]=-2.0$, -1.0 , and -0.5 . We discuss this dwarf, as well as surrounding HST pointings, in some detail in Section 5.4 and in Figures 13 and 14.

brighter (after factoring in the increased distance for Dw7, as well), but the Magellan estimates had large uncertainties at the outset. Dw1 is an extreme case $\left(M_{V} \sim-11\right.$ from ground-based photometry and a revised value of $M_{V} \sim-14$ from HST): we discovered that, at the position of this dwarf, the automated Megacam pipeline provided a significant overestimation in the sky value owing to the large size of Dw1. The sky subtraction in the resulting stacked images was thus excessive and led us to measure a lower total luminosity and surface brightness (the central surface brightness was inferred from integrated light, and the absolute luminosity was derived starting from the central surface brightness value and an exponential profile), which we revise with the HST data set by using resolved stars rather than integrated light. We note that Dw1's structural parameters and luminosity are now in agreement with the analysis of Müller et al. (2017) as well. Dw2 (at only 3! from Dw1) was similarly affected, although to a lesser extent.

\subsection{Metallicities}

We estimate the metallicity content of our target dwarfs by assuming that they host predominantly old and coeval populations, which holds true in the absence of significant young and/ or intermediate-age populations (e.g., bright main sequence and/or AGB stars). Under this assumption, the primary driver of the RGB's color is its intrinsic metallicity, which can thus be computed to first order with photometric information alone. This method is robust for predominantly old populations: the difference between mean spectroscopic and mean photometric metallicities is only $\sim 0.1$ dex for old Local Group dwarfs (e.g., Lianou et al. 2011); differences of up to $\sim 0.5$ dex are observed in the case of prolonged star formation histories. For each galaxy, we derive photometric metallicities for each RGB star brighter than $\mathrm{F} 814 \mathrm{~W}_{0}=25.5$ (where photometric errors are smaller than $\sim 0.15$ mag and the isochrones are most separated in color) by interpolating between Dartmouth isochrones (Dotter et al. 2008) with a fixed age of $10 \mathrm{Gyr}$ and solar-scaled ([ $\alpha /$ $\mathrm{Fe}]=0$ ) metallicities in the range $[\mathrm{Fe} / \mathrm{H}]=-2.5$ to $=-0.5$ (for more details, see Crnojević et al. 2010, 2013). While the choice of age is arbitrary, a slightly younger age would not have a major impact on our results (e.g., adopting $8 \mathrm{Gyr}$ isochrones would change the median metallicity values by $~ 5 \%$; Crnojević et al. 2010); moreover, deep photometric studies of Local Group dwarfs confirm that they all contain old populations (e.g., Weisz et al. 2014). We take into account the foreground/background contamination for the resulting metallicity distribution functions (MDFs) by subtracting the "MDFs" obtained for stars in the 

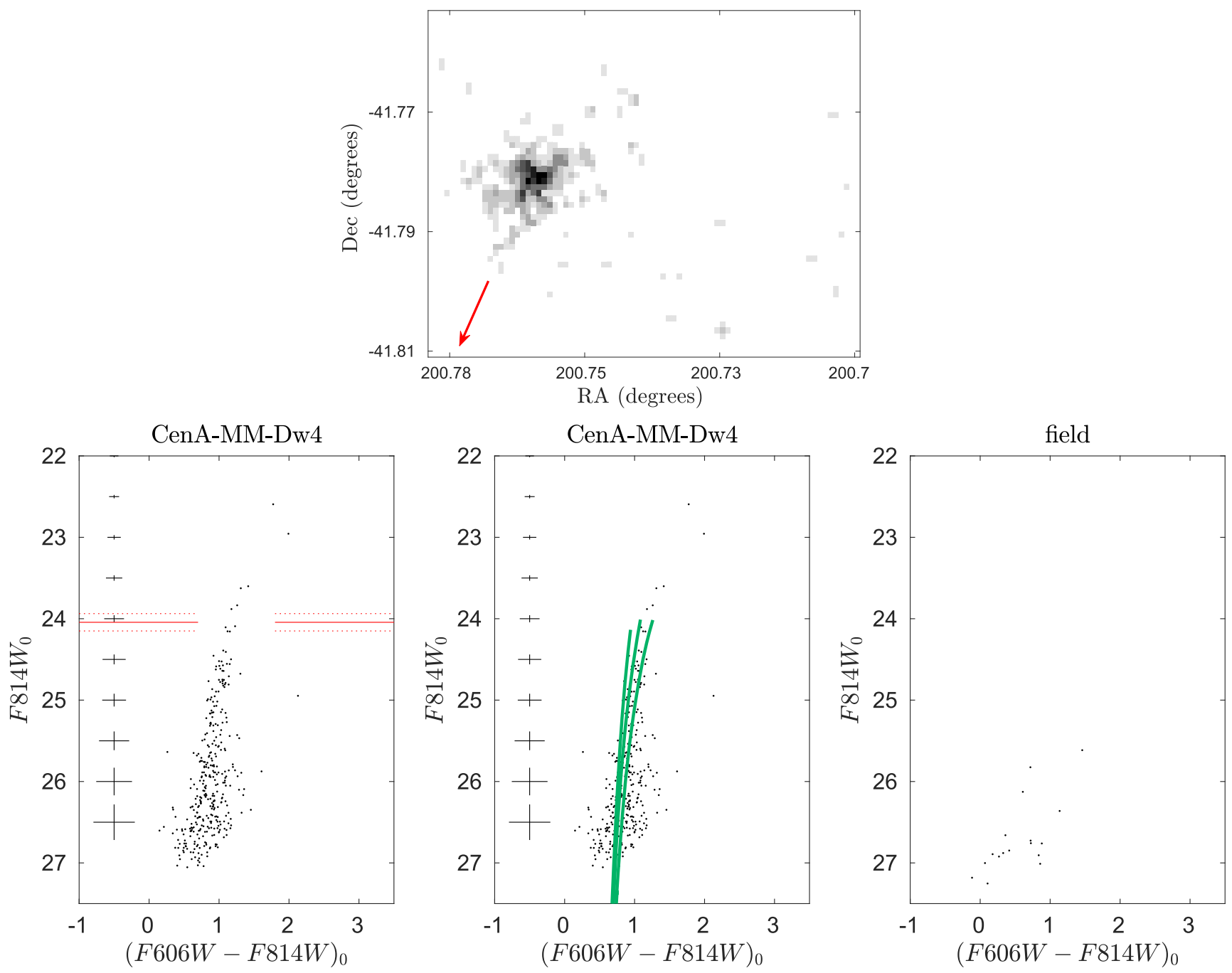

Figure 7. Same as Figure 5, but for Dw4. Stars within $r_{h}$ are shown in the CMD, and isochrones have metallicities of $[\mathrm{Fe} / \mathrm{H}]=-2.5,-1.5$, and -1.0 .

same CMD space (which, in the case of foreground stars, are not necessarily RGB stars) of a spatial region next to each dwarf.

The resulting median metallicities are reported in Table 4. For almost all our targets, the MDFs are well approximated by a Gaussian, with the exception of Dw3, which we further discuss below. The Cen A satellites overall follow the luminosity-metallicity relation defined by Local Group dwarfs (see McConnachie 2012), as shown in Figure 12.

\section{Comments on Individual Dwarfs}

\subsection{An Ultradiffuse Galaxy and Its Possible Companion: Dw1 and Dw2}

Dw1 and Dw2 were first presented in Crnojević et al. (2014b), and due to their projected separation of only 3 !, they appeared like a possible pair of Cen A satellites. In light of their revised distances (Table 4), Dw2 seems to be located $\sim 200 \mathrm{kpc}$ farther away than Dw1, but the values are actually consistent within the error bars. While their distance from each other is unconstrained and we cannot confirm that they are physically bound, we stress that the probability of finding two dwarfs with such a small projected distance over our survey area is negligible.
Dw1 is a very intriguing galaxy on its own: it can be classified as an ultradiffuse galaxy (given the definition of van Dokkum et al. 2015; see also Sandage \& Binggeli 1984 for an early example of this type of galaxy). The exquisite HST imaging allowed us to identify not only a central star cluster with $M_{V} \sim-9$ but also a system of three globular clusters within Dw1's half-light radius (with $-7 \lesssim M_{V} \lesssim-8$ ), all partially resolved into stars (a thorough search for globular clusters around all of the PISCeS dwarf discoveries will be presented in a future work). Spectroscopic follow-up of the clusters has been obtained (A. Seth et al. 2019, in preparation), and it will shed light on the properties of one of the closest ultradiffuse galaxies (e.g., Beasley \& Trujillo 2016; Amorisco et al. 2018; Bennet et al. 2018; Lim et al. 2018). Dw1's properties are overall reminiscent of the Fornax dwarf spheroidal in the Local Group.

For our analysis of Dw1's populations, we combine the Dw1 and Dw2 parallel WFC3 pointings to estimate the foregound/ background contamination. Based on the non-negligible presence of AGB stars above the TRGB with $\mathrm{F} 14 \mathrm{~W}_{0} \lesssim 23.2$, Dw1 is likely to have formed stars until $\sim 1-2$ Gyr ago. We note that a more detailed analysis of the fraction/ages/metallicities of AGB stars in Cen A satellites is rendered difficult by the heavy contamination from foreground MW stars in the same CMD region: a proper decontamination will require near-infrared 

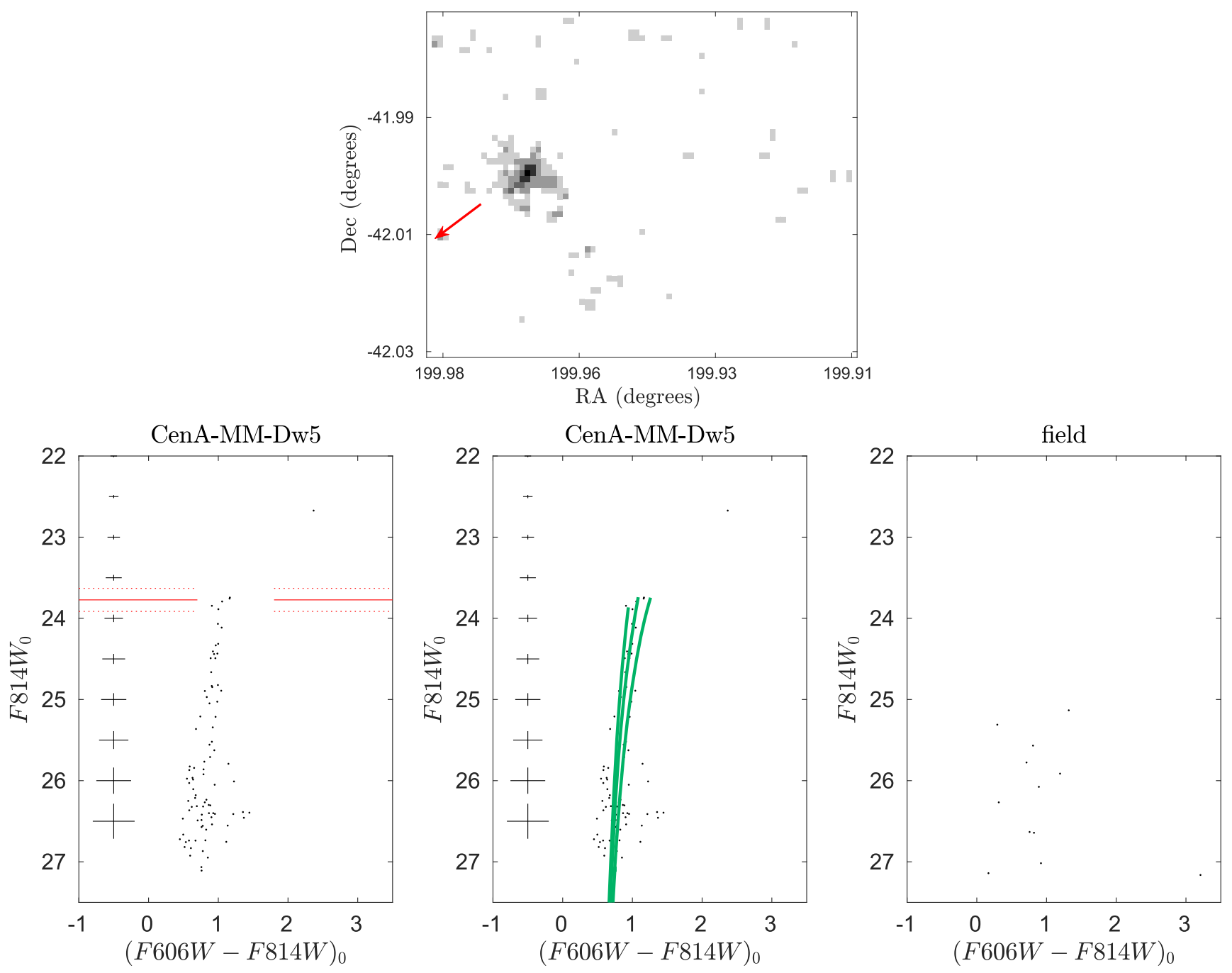

Figure 8. Same as Figure 7, but for Dw5.

imaging, which allows for a clean separation of foreground sequences from stars at the distance of Cen A (see Crnojević et al. 2011). Despite this extended star formation history, Dw1 does not currently contain any gas reservoir (see Table 4), as also confirmed by the absence of young stellar populations in its CMD. The RGB stellar density map from our Magellan imaging (the dwarf extends beyond the HST FOV) does not reveal any asymmetries, despite Dw1's non-negligible elongation $(\epsilon \sim 0.2)$, suggesting that this satellite has not been significantly perturbed by Cen A. Its median metallicity is in line with that expected from the luminosity-metallicity relation derived for Local Group galaxies (McConnachie 2012, their Figure 12). We additionally derive the median metallicity within and beyond its half-light radius, obtaining $[\mathrm{Fe} / \mathrm{H}] \sim-0.99$ for the former and $\sim-1.07$ for the latter and thus revealing a mild metallicity gradient (uncertainties on the derived median metallicities are $\sim 0.01$ dex). Gradients in dwarfs are routinely observed, and they correlate well with galaxy luminosity (e.g., Leaman et al. 2013). More metal-rich (and likely younger) populations are more centrally concentrated, as is the case also for previously known Cen A satellites (see Crnojević et al. 2010, and references therein); this agrees well with the presence of intermediate-age AGB stars in Dw1.
Dw2 contains predominantly old populations (Figure 5), although a few candidate $\mathrm{AGB}$ stars $\left(\mathrm{F} 814 \mathrm{~W}_{0} \lesssim 23.5\right)$ are observed above its TRGB within its half-light radius, pointing to a likely star formation episode between $\sim 2$ and 4 Gyr ago. The foregound/background contamination for this satellite has been estimated from within the ACS FOV, which still contains the outskirts of Dw1. Despite its proximity to the latter, the RGB population of Dw2 shows a regular and rather circular shape, and its median metallicity is consistent with Dw2's absolute magnitude. Finally, the HIPASS upper limit on an H I reservoir in Dw1 only weakly constrains its gas richness.

\section{2. $D w 4, D w 5, D w 6$}

These three faint satellites of Cen A, all located to its north, have a rather large galactocentric distance $(85-125 \mathrm{kpc})$. Due to this, the CMD contamination for the equivalent area covered by each dwarf is negligible (see Figures 7-9). All three dwarfs have well-defined old RGB sequences; Dw4 and Dw6 additionally present a handful of bright sources at $\mathrm{F} 814 \mathrm{~W}_{0} \lesssim 23.6$, which may indicate a low level of star formation between 2 and $4 \mathrm{Gyr}$ ago. Once again, HIPASS only returns weak upper limits on their neutral gas content. Among the three, Dw4 has the highest ellipticity $(\epsilon \sim 0.3)$; however, there is no significant presence of 

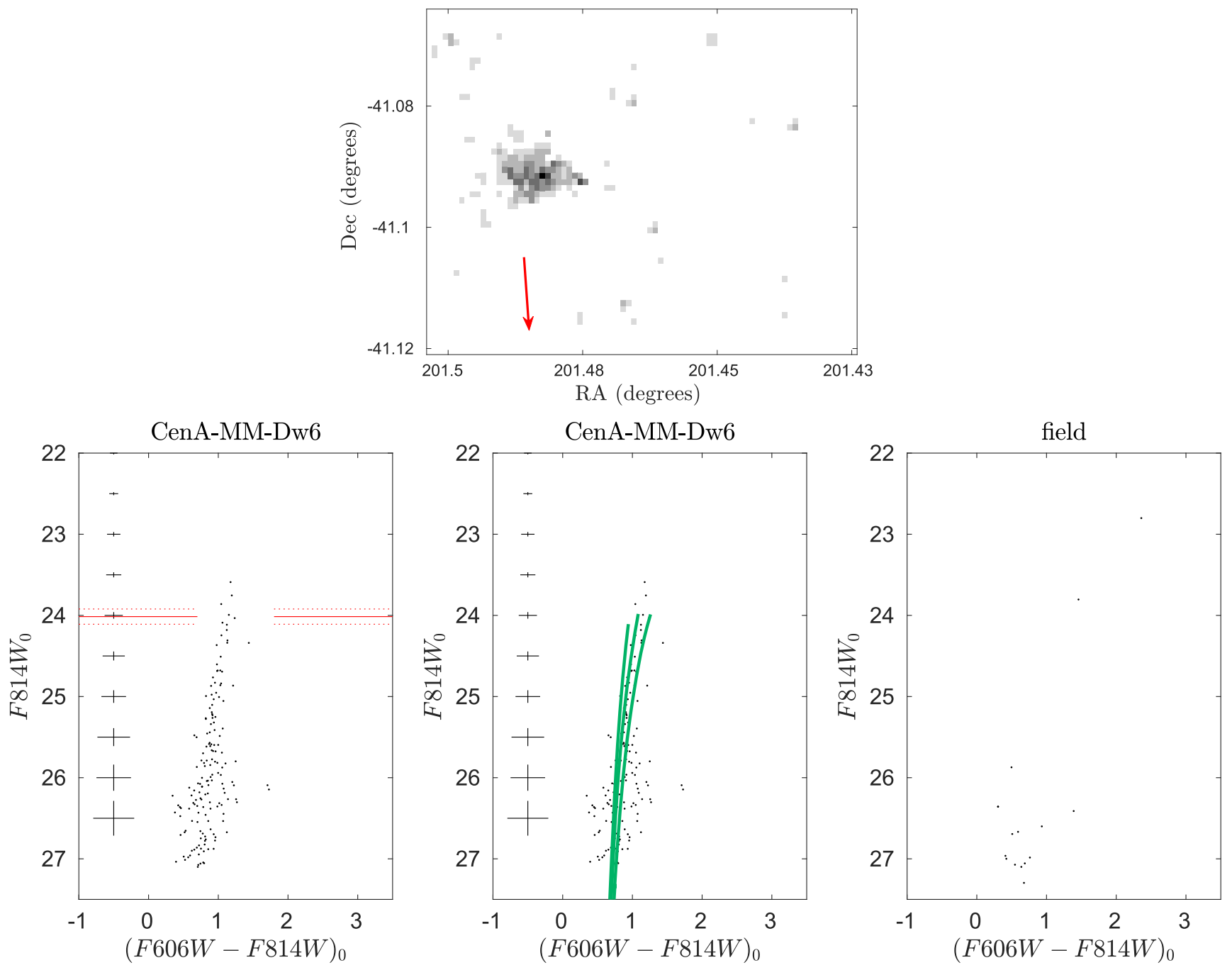

Figure 9. Same as Figure 7, but for Dw6.

debris that could point to tidal disruption for this dwarf. The faintest dwarf uncovered by PISCeS, Dw5, has an unperturbed appearance. Dw6's RGB map shows a very small overdensity in its outskirts to the west, which was already visible in the Magellan data set; this may be connected to Dw6, or may simply represent a background fluctuation. Finally, Dw4 and Dw6 have a median metallicity that places them slightly above, but consistent with, the locus of Local Group dwarfs in the luminosity-metallicity relation.

\subsection{Dw7: A Possible Disruption?}

Due to its proximity to Cen A ( $\sim 40 \mathrm{kpc}$ in projection), the CMD of Dw7 is heavily contaminated by metal-rich populations (the "field" CMD shown in Figure 10 is extracted from the WFC3 parallel pointing). This metal-poor dwarf does not appear to host any young/intermediate-age populations, or detectable neutral gas.

From the RGB density map of Dw7, a small overdensity appears in the NW corner of our ACS pointing: the overdensity is $\sim 8 \sigma$ above the mean stellar density in the lower half of the ACS pointing (which for convenience we call the "primary field"). For reference, the number of Dw7 RGB stars within $1 r_{h}$ is $\sim 60 \sigma$ above the "primary field" level. Interestingly, at the same time the RGB density in the Dw7 WFC3 parallel pointing (or "parallel field") is $\sim 6 \sigma$ below that in the "primary field." We reconsider our ground-based RGB density map in the Dw7 region (top right panel of Figure 10, where we overlay the location of our HST pointings): also within this catalog the RGB overdensity is visible and detected at a $\sim 5 \sigma$ level. However, there is virtually no difference in number counts between the "primary field" and "parallel field" in the Magellan photometry. This may be attributed to the overall low number of RGB stars ( $\sim 40$ in Magellan vs. the $\sim 350$ in the ACS pointing, for the "primary field"): the significantly deeper HST images allow a cleaner selection of RGB stars and unveil features that were not detected in the ground-based photometry. Given the small size of the HST pointings, it is not possible to investigate the presence of further overdensities around Dw7 from this data set; a closer look at the ground-based map does not highlight unambiguous asymmetries/tails emanating from Dw7, as the area around this dwarf is heavily contaminated by Cen A's halo stars and presents several randomly distributed overdensities. Finally, the radial RGB density profile of Dw7 does show an excess of sources at large galactocentric radii with respect to a simple exponential profile (the uncertainties on the structural parameters are indeed higher than for the other dwarfs; see Table 4). The lower stellar density found in the WFC3 pointing might imply 

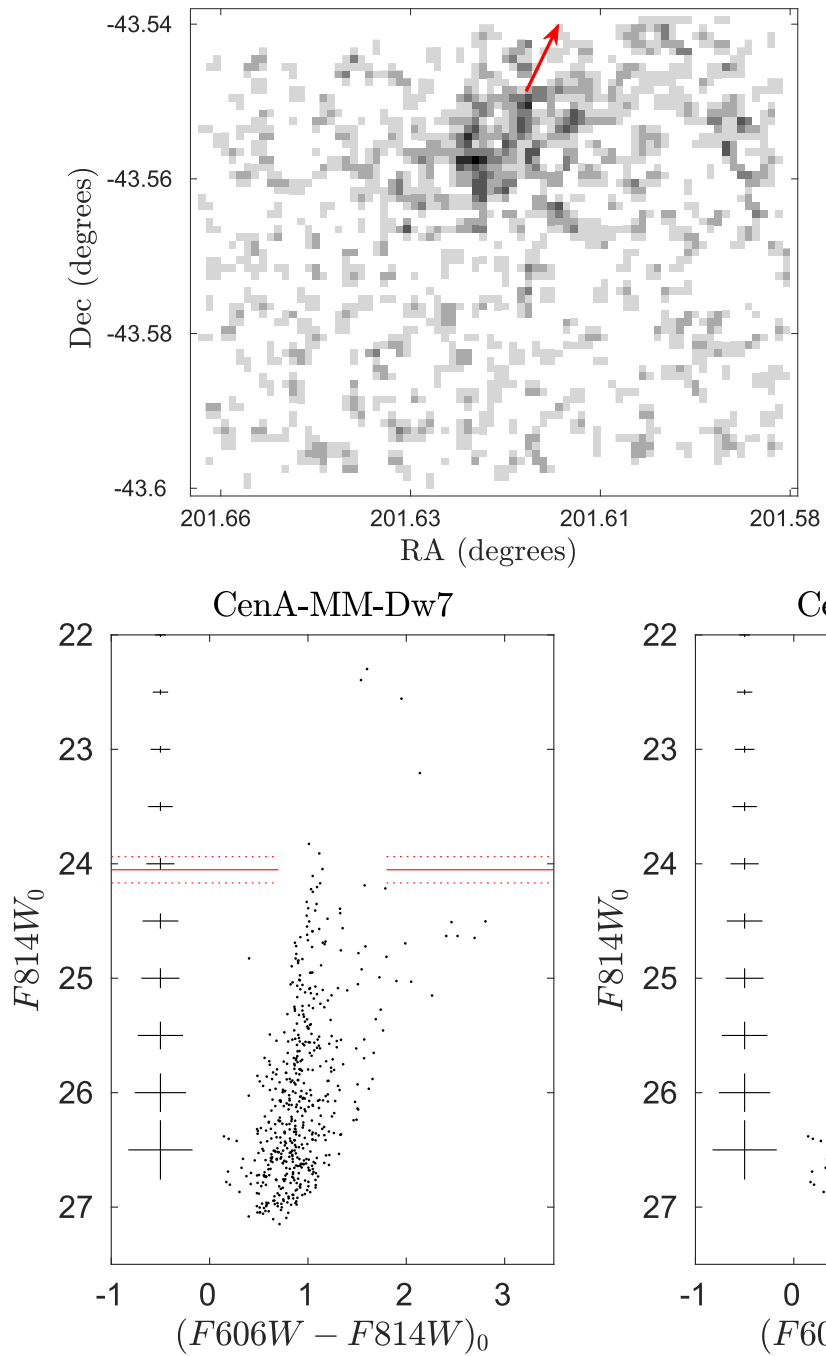
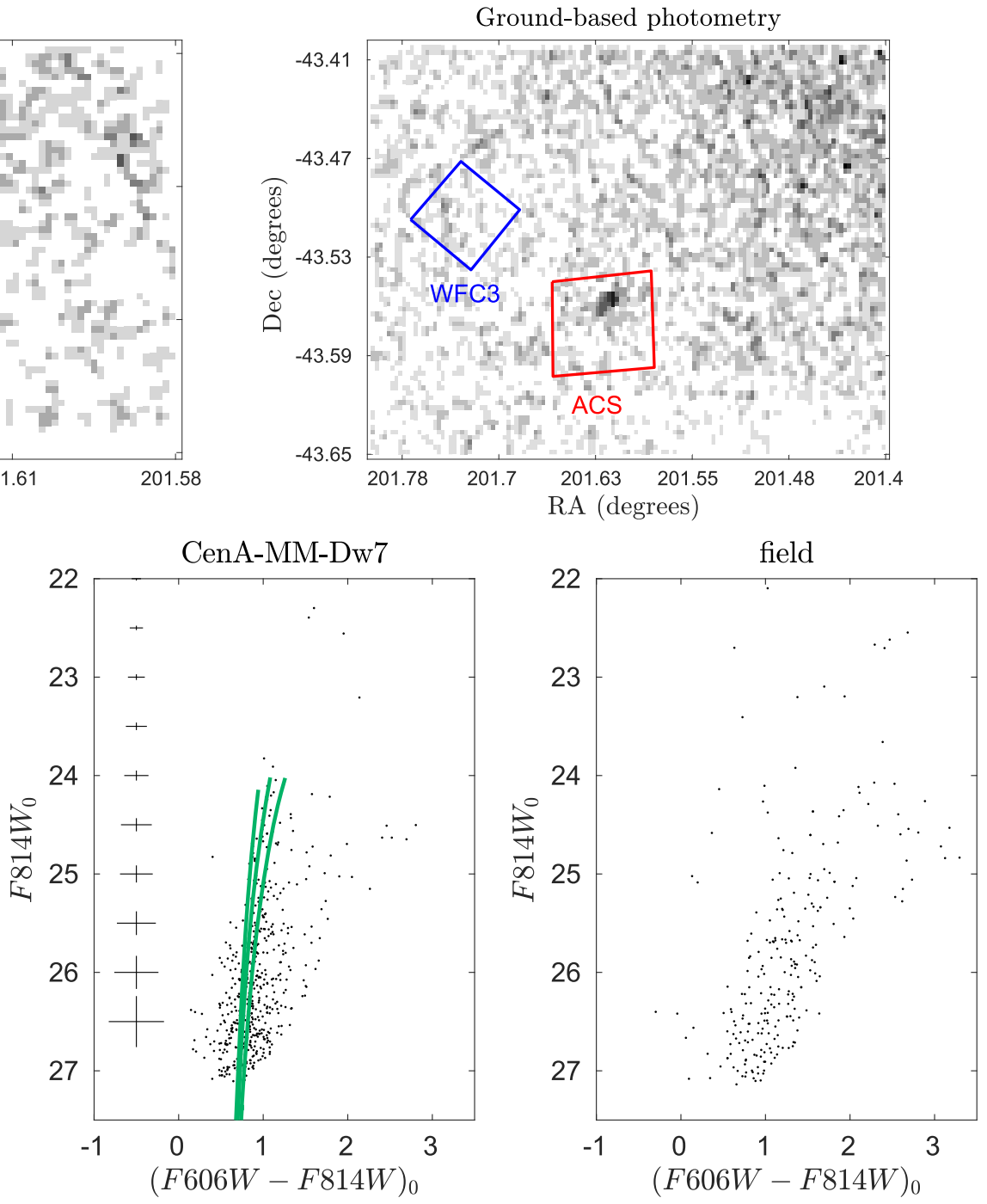

Figure 10. Same as Figure 7, but for Dw7. The additional RGB spatial density map (top right panel) is derived from our ground-based Magellan photometry, and the positions of the ACS and WFC3 pointings are overlaid (see Section 5). The contamination from Cen A's metal-rich stellar populations at the position of Dw7 is clear from both the RGB density map and the field CMD.

Table 3

Properties of Unresolved Background Galaxies

\begin{tabular}{|c|c|c|c|}
\hline Parameter & Dw10-C16 & Dw11-C16 & Dw12-C16 \\
\hline R.A. (h:m:s) & $13: 26: 49 " .4 \pm 1 " 52$ & $13: 21: 40 " ! 3 \pm 0 " 198$ & $13: 24: 10^{\prime \prime} 9 \pm 1{ }^{\prime \prime} 00$ \\
\hline Decl. (d:m:s) & $-43: 00: 01 \pm 1$ ". 46 & $-43: 05: 00 \pm 1 ! 08$ & $-42: 08: 24 \pm 9 ! \prime 00$ \\
\hline$m_{g}(\mathrm{mag})$ & $21.9 \pm 0.3$ & $20.8 \pm 0.4$ & $19.3 \pm 0.1$ \\
\hline$m_{r}(\mathrm{mag})$ & $21.3 \pm 0.4$ & $19.6 \pm 0.5$ & $18.8 \pm 0.2$ \\
\hline$r_{h}(\operatorname{arcsec})$ & $3.93 \pm 0.97$ & $11.70 \pm 3.46$ & $7.23 \pm 0.69$ \\
\hline$n$ (Sérsic index) & $0.53 \pm 0.17$ & $0.42 \pm 0.18$ & $0.62 \pm 0.05$ \\
\hline$\epsilon$ & $0.36 \pm 0.10$ & $0.68 \pm 0.10$ & $0.36 \pm 0.04$ \\
\hline
\end{tabular}

that the ACS FOV contains a lingering low-density stellar contribution from Dw7, even though our data are not deep enough to confirm an ongoing disruption.

\subsection{The Curious Case of Dw3: Population Gradients along Its Tails}

The largest coherent substructure in the halo of Cen A is constituted by the disrupting Dw3 and its extended (more than
1 deg) tidal stream. The outer isophotes in the dwarf's remnant show an S shape typical of tidal disruption (see Crnojević et al. 2016a), although on a larger scale than the ACS FOV. Dw3 additionally hosts an elongated central star cluster (A. Seth et al. 2019, in preparation), which is partially resolved into stars in the HST imaging.

The CMD for the inner region of Dw3's remnant is presented in Figure 6, and it shows a relatively broad RGB and a luminous AGB extending to colors even redder than that of the TRGB. The 
Table 4

Properties of the Confirmed Cen A Satellites

\begin{tabular}{|c|c|c|c|c|c|c|c|}
\hline Parameter & Dw1 & Dw2 & Dw3 & Dw4 & Dw5 & Dw6 & Dw7 \\
\hline R.A. (h:m:s) & $13: 30: 14 " .31 \pm 1{ }^{\prime \prime} 52^{\mathrm{a}}$ & 13:29:57!" $42 \pm 0$ "!98 & 13:30:20" $44 \pm 1 . " 00$ & $13: 23: 02 "$ "56 \pm 0 ". 55 & $13: 19: 52 "$ " $42 \pm 0$ ".79 & $13: 25: 57$ !" $25 \pm 1$ 1" 15 & $13: 26: 28 . " 55 \pm 1$ " 82 \\
\hline Decl. (d:m:s) & $-41: 53: 34 ! \cdot 78 \pm 1$ "' $46^{\mathrm{a}}$ & $-41: 52: 23 " \prime 70 \pm 1 " \prime 08$ & $-42: 11: 30 ! 27 \pm 11 ! " 00$ & $-41: 47: 08.95 \pm 0 . \prime 61$ & $-41: 59: 40 " \prime 68 \pm 0 . " 65$ & $-41: 05: 37 ! " 13 \pm 0$ ". 80 & $-43: 33: 23 ! .07 \pm 1 ! 78$ \\
\hline$E_{(B-V)}$ & 0.11 & 0.11 & 0.10 & 0.11 & 0.11 & 0.10 & 0.10 \\
\hline$(m-M)_{0}(\mathrm{mag})$ & $27.96 \pm 0.07$ & $28.09 \pm 0.12$ & $27.94 \pm 0.09$ & $28.06 \pm 0.14$ & $27.79 \pm 0.19$ & $28.03 \pm 0.11$ & $28.07 \pm 0.15$ \\
\hline$D(\mathrm{Mpc})$ & $3.91 \pm 0.12$ & $4.14_{-0.22}^{+0.24}$ & $3.88_{-0.15}^{+0.16}$ & $4.09_{-0.25}^{+0.26}$ & $3.61_{-0.31}^{+0.34}$ & $4.04_{-0.19}^{+0.20}$ & $4.11_{-0.27}^{+0.29}$ \\
\hline$D_{\text {CenA,proj }}(\mathrm{deg})$ & 1.43 & 1.42 & 1.22 & 1.31 & 1.45 & 1.93 & 0.57 \\
\hline$D_{\mathrm{CenA}, \mathrm{proj}}(\mathrm{kpc})$ & 93 & 92 & 79 & 85 & 94 & 125 & 37 \\
\hline$\epsilon$ & $0.22 \pm 0.02^{\mathrm{b}}$ & $<0.17$ & $0.29 \pm 0.19^{\mathrm{a}, \mathrm{b}}$ & $0.32 \pm 0.05$ & $<0.20$ & $0.25 \pm 0.08$ & $0.41 \pm 0.08$ \\
\hline P.A. $\left(\mathrm{N}\right.$ to $\left.\mathrm{E} ;{ }^{o}\right)$ & $51.1 \pm 6.1^{\mathrm{a}}$ & $\ldots$ & $\ldots$ & $-36.8 \pm 4.3$ & $\ldots$ & $86.9 \pm 9.5$ & $-46.1 \pm 6.5$ \\
\hline$r_{h}(!)$ & $1.60 \pm 0.03^{\mathrm{a}}$ & $0.34 \pm 0.03$ & $2.21 \pm 0.15^{\mathrm{a}, \mathrm{b}}$ & $0.33 \pm 0.01$ & $0.18 \pm 0.01$ & $0.26 \pm 0.01$ & $0.50 \pm 0.05$ \\
\hline$r_{h}(\mathrm{kpc})$ & $1.82 \pm 0.03$ & $0.41 \pm 0.04$ & $2.49 \pm 0.17$ & $0.39 \pm 0.01$ & $0.19 \pm 0.01$ & $0.31 \pm 0.01$ & $0.60 \pm 0.06$ \\
\hline$\mu_{V, 0}\left(\operatorname{mag} \operatorname{arcsec}^{-2}\right)$ & $24.7 \pm 0.2$ & $25.8 \pm 0.4$ & $26.0 \pm 0.4$ & $25.1 \pm 0.2$ & $25.6 \pm 0.3$ & $25.4 \pm 0.3$ & $25.9 \pm 0.4$ \\
\hline$M_{V}(\mathrm{mag})$ & $-13.8 \pm 0.1$ & $-9.7 \pm 0.2$ & $-13.1 \pm 0.1^{\mathrm{c}}$ & $-9.9 \pm 0.2$ & $-8.2 \pm 0.2$ & $-9.1 \pm 0.2$ & $-9.9 \pm 0.3$ \\
\hline$L_{*}\left(10^{5} L_{\odot}\right)$ & $283.1 \pm 26.1$ & $6.5 \pm 1.2$ & $148.6 \pm 13.7$ & $7.8 \pm 1.4$ & $1.6 \pm 0.3$ & $3.7 \pm 0.7$ & $7.8 \pm 2.2$ \\
\hline$M_{\mathrm{H} \mathrm{I}}{ }^{\mathrm{d}}\left(10^{6} M_{\odot}\right)$ & $\lesssim 5.5$ & $\lesssim 6.2$ & $\lesssim 4.3$ & $\lesssim 5.1$ & $\lesssim 3.8$ & $\lesssim 4.6$ & $\lesssim 6.8$ \\
\hline$M_{\mathrm{H} \mathrm{I}} / L_{*}\left(M_{\odot} / L_{\odot}\right)$ & $\lesssim 0.2$ & $\lesssim 9.5$ & $\lesssim 0.3$ & $\lesssim 6.6$ & $\lesssim 23.4$ & $\lesssim 12.5$ & $\lesssim 8.8$ \\
\hline$[\mathrm{Fe} / \mathrm{H}]_{\text {med }}$ & $-1.02 \pm 0.01$ & $-1.58 \pm 0.07$ & $-0.61 \pm 0.01^{\mathrm{c}}$ & $-1.15 \pm 0.01$ & $-1.46 \pm 0.02$ & $-1.20 \pm 0.01$ & $-1.47 \pm 0.05$ \\
\hline
\end{tabular}

Notes.

a These values have been derived starting from our Magellan/Megacam photometry (the HST data do not cover the entire extent of these galaxies).

${ }^{\mathrm{b}}$ From Crnojević et al. (2016a); this is an indicative value only, since the studied galaxy is being heavily disrupted.

${ }^{\mathrm{c}}$ Excluding tidal tails.

${ }^{\mathrm{d}} 5 \sigma$ upper limits from HIPASS. 

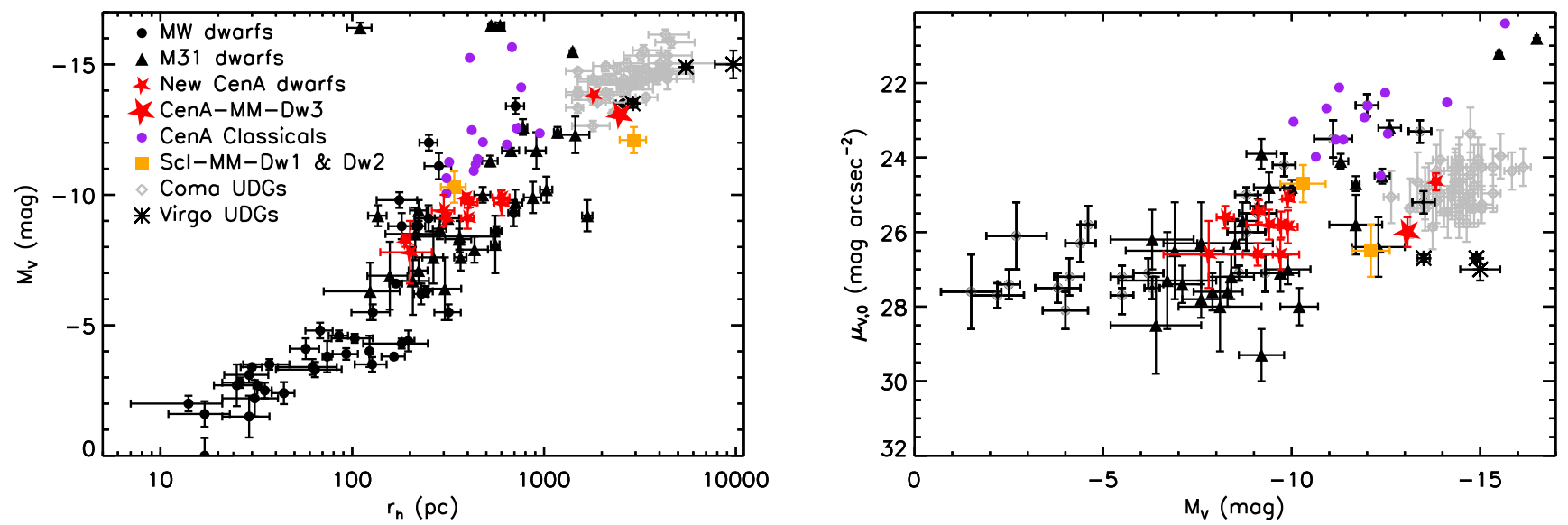

Figure 11. Properties of the new Centaurus A satellites with respect to Local Group and other galaxy samples. Left panel: absolute $V$-band magnitude as a function of half-light radius. Right panel: central surface brightness as a function of absolute magnitude. The Cen A dwarfs presented in this work, with updated physical properties, are shown as red stars. The tidally disrupting CenA-MM-Dw3 is denoted with a larger red star symbol. The general properties of the faint Cen A dwarfs presented here are consistent with analogous galaxies in the Local Group. They are also fainter and have a lower surface brightness than the previously know Cen A sample. CenA-MM-Dw1 and the disrupting dwarf CenA-MM-Dw3 are comparable to ultradiffuse galaxies seen in the Virgo and Coma Clusters. The data for both panels come from MW and M31 dwarf galaxies (black circles and triangles, respectively; data from McConnachie 2012; Sand et al. 2012; Crnojević et al. 2014a; Drlica-Wagner et al. 2015; Kim et al. 2015; Kim \& Jerjen 2015; Koposov et al. 2015; Laevens et al. 2015a, 2015b; Martin et al. 2015; Crnojević et al. 2016a; DrlicaWagner et al. 2016; Torrealba et al. 2016; Carlin et al. 2017; Koposov et al. 2018; Mutlu-Pakdil et al. 2018; Torrealba et al. 2018), recently discovered PISCeS dwarfs in NGC 253 (orange squares; Sand et al. 2014; Toloba et al. 2016), diffuse galaxies in Virgo and Coma (black asterisks and gray diamonds, respectively; Mihos et al. 2015; van Dokkum et al. 2015), and previously known "classical" Cen A dwarfs (purple circles; Sharina et al. 2008).

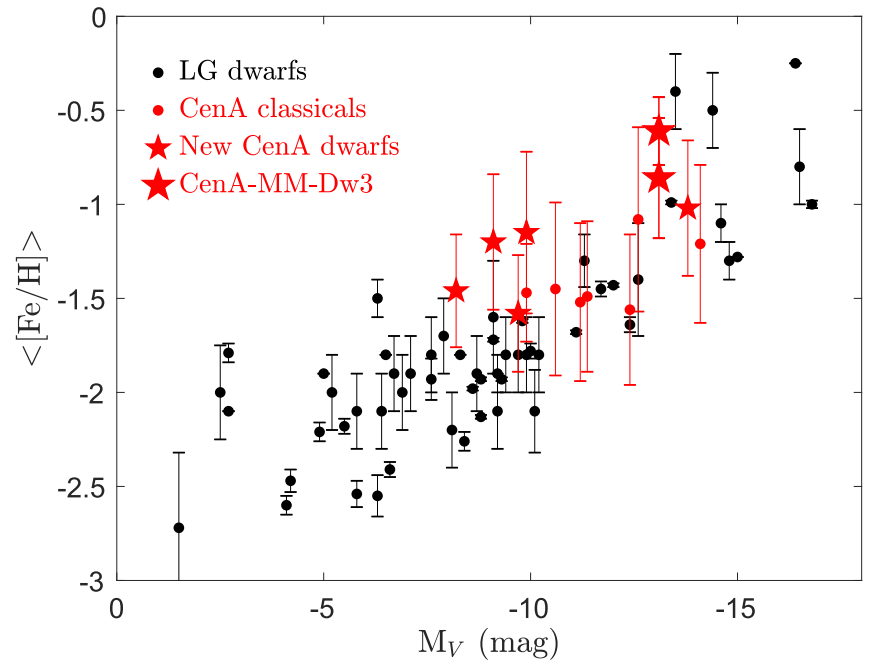

Figure 12. Mean/median metallicity vs. absolute magnitude for Local Group dwarfs and Cen A satellites, respectively. The values for Local Group dwarfs are taken directly from McConnachie (2012) and are derived with a broad range of techniques. The "classical" Cen A dwarfs are those studied in Crnojević et al. (2010), for which metallicities are derived with the same photometric procedure as in this study (the other Cen A satellites do not have reliable metallicity measurements to the best of our knowledge). For Dw3, we report median metallicities for both its remnant and the most metal-poor pointing along its tail (Dw3S; see Figure 13). The error bars for the PISCeS dwarfs' metallicities represent the Gaussian spreads of their MDFs.

excess of AGB stars at $F 814 W_{0} \lesssim 23.1$ with respect to the foreground/background rescaled field (which is extracted from the Dw3S parallel pointing, see below) suggests a prolonged star formation, possibly until $\sim 1-2 \mathrm{Gyr}$ ago as indicated by the luminosity of the brightest AGB stars; later star formation is unlikely given the lack of blue sequences in Dw3's CMD. The HST data set allows us to revise the distance of Dw3 and to place it at the same distance as Cen A. As in Crnojević et al. (2016a), we repeat the measurement of the RGB stars' luminosity in the tails of Dw3 from our Megacam data (see Crnojević et al. 2016a), adopting the new distance estimate, and we confirm that the original total magnitude of Dw3 (i.e., prior to tidal disruption) could have been as bright as $M_{V} \sim-15$. This can be further established from the median metallicity of Dw3's remnant, which would place it at a comparable absolute magnitude in Figure 12.

In Figure 13 we show a zoom-in RGB stellar density map around the remnant of Dw3: the map is derived from our ground-based Magellan imaging, and overlaid are the positions of our follow-up HST pointings in this region (Dw1, Dw2, Dw3, Dw3S, and their relative parallel fields), along with one nearby pointing and its parallel, which we dub Dw3N, from program GO-12964 (PI Rejkuba; see Rejkuba et al. 2014; these fortuitously lay on top of Dw3's tidal tail). In the subsequent analysis, we will adopt the Dw3S parallel WFC3 pointing as the "field" for Dw3, since it is located off of the tail. In Figure 13 we further report the CMDs for the HST pointings in this area, in order to look for possible gradients in the distances/stellar populations along Dw3's tails.

We first compute the TRGB distance for each pointing (as described in Section 4.3), which we report in Table 5, while the TRGB magnitudes are overplotted on each CMD. Overall, there is good agreement between the distances along the tails and that of Dw3's remnant within the uncertainties, and there is no strong indication of a distance gradient. The only discrepant pointing is the parallel of Dw3 (Dw3-WFC3), which seems to be $\sim 600 \mathrm{kpc}$ more distant: given that the Dw3S pointing, at a larger Dw3-centric distance, has a distance consistent with Dw3, we suspect that the Dw3-WFC3 distance might be due to a combination of small number statistics and small-scale inhomogeneities in the stream populations. For the rest of this analysis, we will adopt Dw3's nominal distance for all the pointings.

In the bottom of Figure 13 we also present an RGB radial density profile as a function of distance from Dw3: also here, Dw3-WFC3 seems to contain fewer stars than expected from the overall profile. It is worth noting that the orientation and the shape of Dw3's stream are hard to reconcile with tidal disruption by Cen A; however, no other nearby galaxy could be responsible 

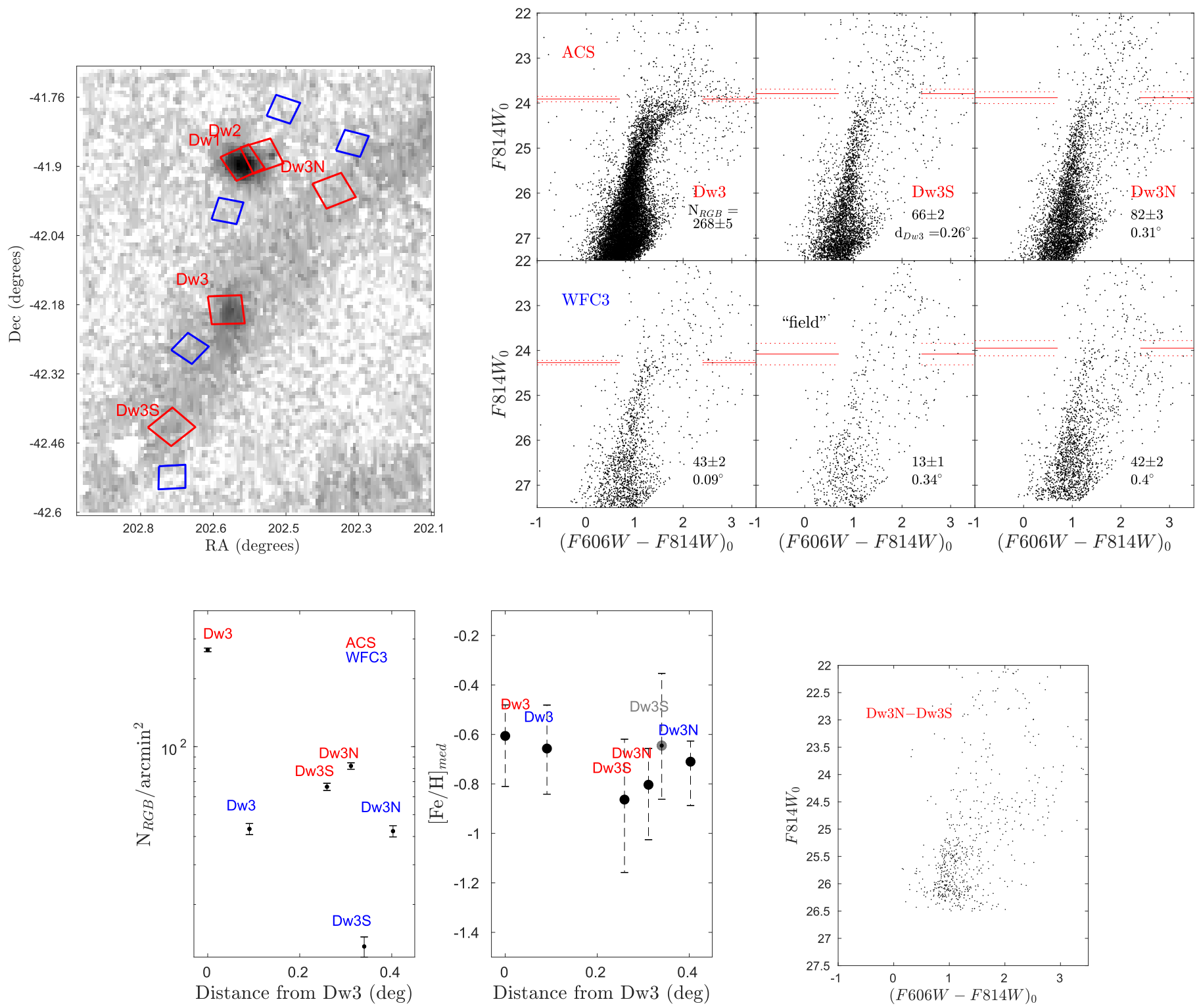

Figure 13. Top left panel: position of the HST fields in the Dw3 area, overlaid on the RGB spatial density map from our ground-based Magellan photometry. Red squares are for the primary ACS/WFC pointings, blue for the parallel WFC3/UVIS ones (the parallel pointing for each primary is the closest blue square to it). Top right panels: CMDs for the HST fields related to Dw3, shown in the top left panel and labeled accordingly (the primary ACS pointings are in the top panels, while the corresponding parallel WFC3 pointings are below them). The number of stars per square! in the RGB selection box is reported for each field, as is the projected distance (in deg) of each pointing from the center of Dw3. The TRGB magnitudes are also reported as red lines. Bottom left and middle panels: number of RGB stars per square! and median metallicity as a function of distance from the center of Dw3, labeled as above. The metallicity error bars denote 50th percentile intervals for the respective MDFs. Bottom right panel: CMD obtained after statistical subtraction of Dw3S pointing stars from Dw3N pointing.

Table 5

Distances and Metallicities along Dw3's Tails

\begin{tabular}{lccc}
\hline \hline Pointing & $(m-M)_{0}(\mathrm{mag})$ & $D(\mathrm{Mpc})$ & {$[\mathrm{Fe} / \mathrm{H}]_{\text {med }}$} \\
\hline Dw3-ACS & $27.94 \pm 0.09$ & $3.88 \pm 0.16$ & $-0.61 \pm 0.01$ \\
Dw3-WFC3 & $28.29 \pm 0.08$ & $4.54 \pm 0.17$ & $-0.66 \pm 0.01$ \\
Dw3S-ACS & $27.81 \pm 0.12$ & $3.65 \pm 0.20$ & $-0.86 \pm 0.01$ \\
Dw3S-WFC3 & $28.10 \pm 0.25$ & $4.16 \pm 0.51$ & $-0.65 \pm 0.01$ \\
Dw3N-ACS & $27.89 \pm 0.14$ & $3.79 \pm 0.25$ & $-0.80 \pm 0.01^{\mathrm{a}}$ \\
Dw3N-WFC3 & $27.97 \pm 0.18$ & $3.92 \pm 0.33$ & $-0.71 \pm 0.02^{\mathrm{a}}$ \\
\hline
\end{tabular}

Note.

a The median metallicity has been derived excluding values with $[\mathrm{Fe} /$ $\mathrm{H}]>-0.5$. for its disruption (see also Crnojević et al. 2016a). What is even more noteworthy is that the stellar density increases again beyond a galactocentric radius of $\sim 0.3 \mathrm{deg}$ (i.e., for the pointings along the northern portion of the stream), instead of continuously decreasing as a function of radius. A similar result using the same HST data was found by Rejkuba et al. (2014, their pointing $\mathrm{F} 6$ ). We investigate this further by inspecting the CMDs of Dw3N and Dw3N-WFC3: they both seem to have an excess of sources with redder colors than the main RGB locus and at magnitudes fainter than the TRGB, when compared to all the other pointings. We perform the following test: we statistically subtract sources of the Dw3S CMD from the Dw3N CMD, given their comparable Dw3-centric distance. We 

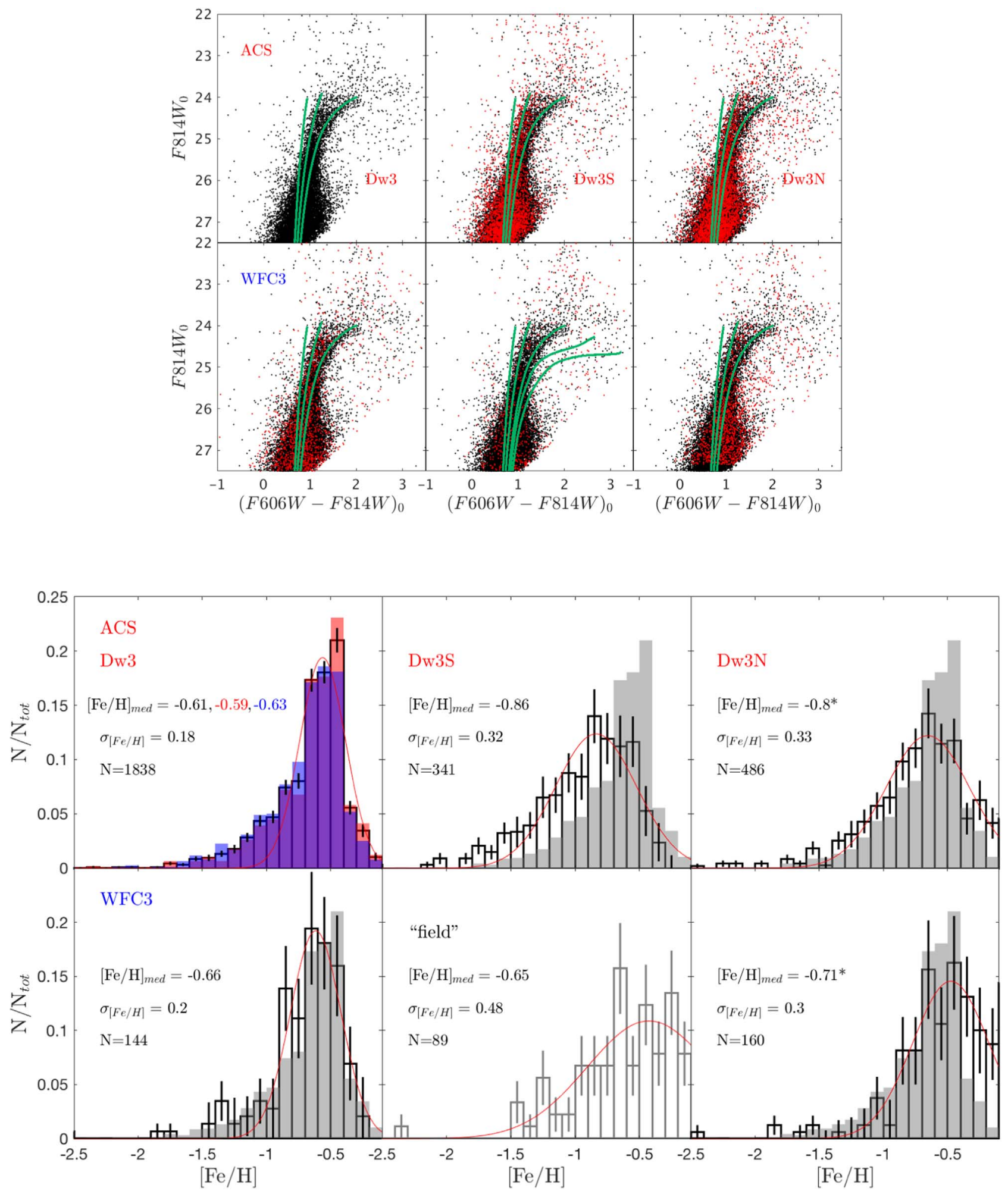

Figure 14. Top panels: same CMDs as in Figure 13, with overlaid $10 \mathrm{Gyr}$ isochrones of varying metallicity $([\mathrm{Fe} / \mathrm{H}]=-2.0,-1.0$, and -0.5 ; plus -0.3 and -0.1 for the bottom middle panel to illustrate the full range of isochrones used to derive photometric metallicities). The underlying black CMD in each subpanel is the one from the central pointing of Dw3 (top left subpanel). Bottom panels: MDFs for each pointing in the top panels, derived by interpolating metallicity values for individual RGB stars between isochrones with fixed age and varying metallicity. The foreground/background contamination has been subtracted using the Dw3S parallel pointing as "field" (bottom middle panel); the MDFs are normalized to the total number of stars for which metallicities have been derived. The median metallicity and the metallicity dispersion from the best-fitting Gaussian (red line) are reported for each field, as is the number of RGB stars included in the MDF. The underlying gray MDF in each subpanel is the one from the central pointing of Dw3 (top left subpanel). For the central Dw3 pointing, we also report the MDFs with the respective median metallicities for stars within (red histogram) and beyond (blue histogram) $0.5 r_{h}$. 
Table 6

Absolute Luminosities of Cen A Group Members within $300 \mathrm{kpc}$ of Cen A; Those Located beyond $150 \mathrm{kpc}$ Are below the Horizontal Line

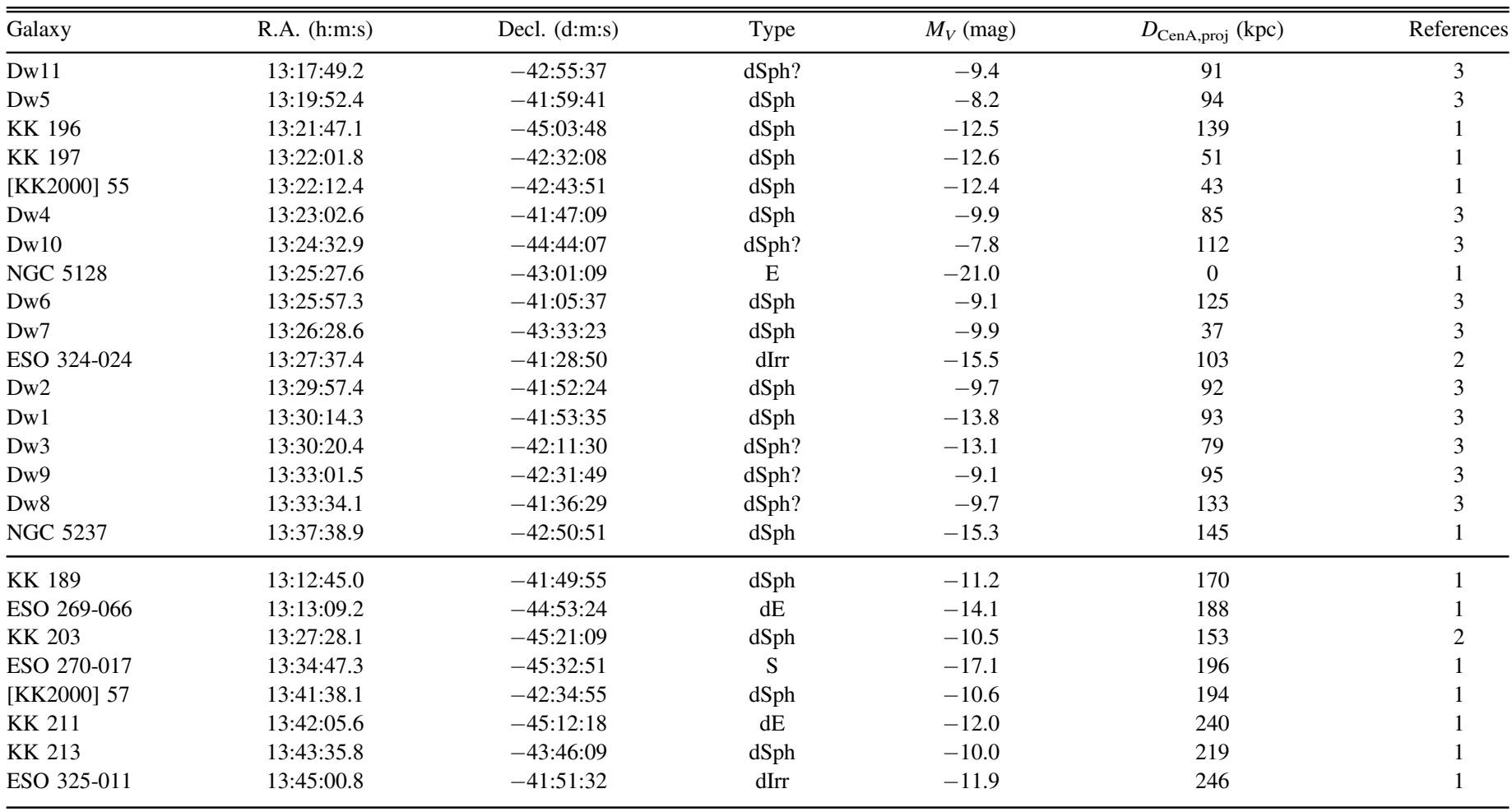

References. (1) Sharina et al. 2008, updated with latest Karachentsev et al. 2013 distance measurements; (2) $M_{B}$ value from Karachentsev et al. 2013, from which $M_{V}$ is estimated as $M_{B}-0.31$ (see text for details); (3) this work.

plot the resulting CMD distribution of one realization of the subtraction in the bottom of Figure 13: the residual sequences resemble a more distant RGB with a possible well-populated luminous AGB extending to red colors. We derive a TRGB value of $\sim 25.4$ for this putative stellar population, which would place it at a distance of $\sim 7.6 \mathrm{Mpc}$. The overdensity along the northern portion of Dw3's stream is clearly visible from our RGB density map, and the overdensity in the CMD space is confirmed by our test: while these are undeniable, the presence of a possible background object at such a large distance is puzzling. Its spatial properties cannot be investigated further (the Magellan data set is too foreground/background contaminated), and a search of the NASA Extragalactic Database (NED) does not reveal any other background galaxies in this area. Even if the observed features were truly part of a background overdensity, it is impossible for this to have been interacting with Dw3 given their relative distances.

To summarize, the northern portion of Dw3's stream presents an overdensity with respect to the expected radial decline (as evinced from the southern portion of the stream; Figure 13), which is possibly due to a distant, resolved background object. Next, we will discuss the implications on the derived MDF of Dw3N.

The shapes of the RGB sequence in the Dw3 pointings differ significantly from each other (Figure 14, top panels), suggesting a varying metallicity content. We derive photometric metallicities as described above and statistically subtract the "field" contribution from the MDFs as derived from the Dw3S-WFC3 pointing, which is dominated by Cen A's halo stars. The resulting MDFs and median metallicities are presented in the bottom panels of Figure 14; for the central Dw3 pointing, we additionally compute the MDFs within and beyond $0.5 r_{h}$. In Figure 13 we also plot the median metallicities as a function of radius (with the relative 50th percentile metallicity intervals). A gradient of $-0.03 \mathrm{dex} \mathrm{kpc}^{-1}$ is present within the central regions of the disrupting dwarf; along the southern portion of the stream the median metallicity decreases significantly $(-0.25$ dex over $\sim 17 \mathrm{kpc}$ ) with respect to Dw3's remnant, as also evidenced by the varying MDF shape between Dw3 and Dw3S (Figure 14). If we extrapolated the inner gradient to the outer regions of Dw3, the Dw3S populations would have originally come from a distance of $\sim 8 \mathrm{kpc}$ from the center, rather than the currently observed $\sim 17 \mathrm{kpc}$. To justify this large Dw3-centric distance, either Dw3 has a past as an extremely diffuse galaxy, or its predisruption metallicity gradient must have been steeper than the one currently observed. The MDFs for the Dw3N pointings seem to contain a metal-rich population, stemming from the red sources identified in their CMDs and discussed above; this metal-rich "tail" is not observed in the Dw3S MDFs. We thus assume these to be residual contaminants after our standard "field" subtraction, and we compute the median metallicities for the Dw3N pointings excluding all metallicities $[\mathrm{Fe} / \mathrm{H}]>-0.5$. The resulting values are still higher than that expected from the gradient found along the southern tail portion (see the metallicity profile in Figure 13), because the distribution of contaminating sources in the CMD is indeed not limited to the reddest colors.

We additionally note that the shape of the Dw3 MDFs is not well approximated by a Gaussian: this is not surprising and already observed in Local Group dwarfs for which spectroscopic MDFs have been derived (Kirby et al. 2013). In particular, the MDF fall-off on the metal-rich end can be explained by the effects of supernova explosions and stellar 

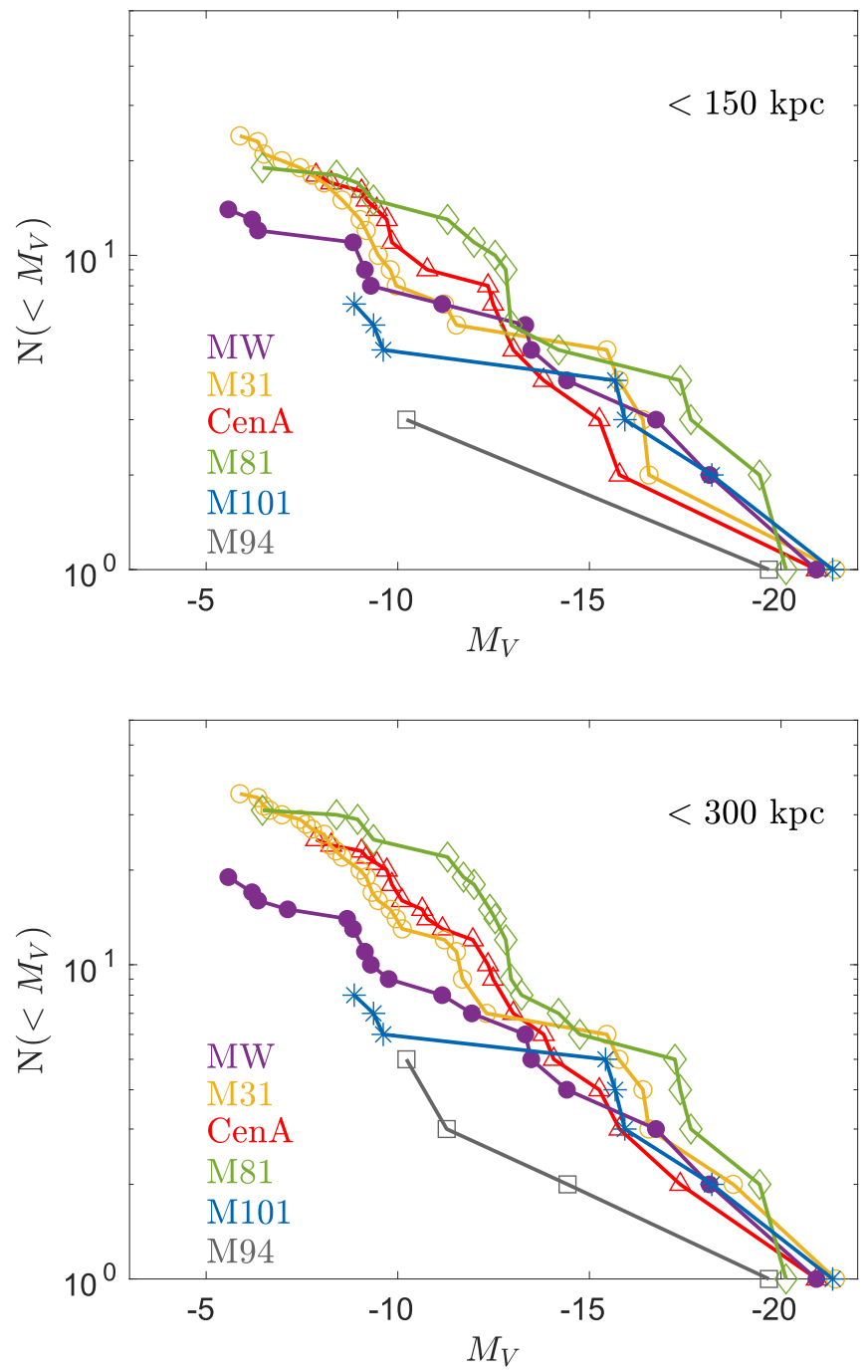

Figure 15. CLFs for Cen A (red triangles and line), the MW (purple circles), Andromeda (yellow circles), M81 (green diamonds), M101 (cyan asterisks), and M94 (gray squares). The top panel shows satellites within a projected radius (or 3D radius for the $\mathrm{MW}$ ) of $150 \mathrm{kpc}$; the bottom panel includes objects within $300 \mathrm{kpc}$ of each host (see text for details). For the latter sample, a cumulative Schechter function gives faint-end slopes $\alpha$ of $-1.14_{-0.16}^{+0.17}$ for Cen $\mathrm{A},-1.13_{-0.08}^{+0.08}$ for the $\mathrm{MW},-1.22_{-0.10}^{+0.11}$ for Andromeda, $-1.06_{-0.12}^{+0.31}$ for M81, -0.80 for M101, and $\sim-1.16$ for M94 (the latter two are poorly constrained).

winds on the evolution of the dwarf (see Crnojević et al. 2010, and references therein). We plan a more in-depth analysis of Dw3's stellar populations via spectroscopy obtained with VLT/VIMOS (E. Toloba et al. 2019, in preparation). Finally, the differences in the MDFs of the Dw3 tails and of the "field" pointing (Figure 14) demonstrate how the material stripped off of this relatively massive dwarf could not be the primary source of populations in the Cen A halo at these distances, since the latter peaks at significantly more metal-rich values (in accordance with the predictions of D'Souza \& Bell 2018; see also Rejkuba et al. 2011, 2014).

\section{Cen A Satellite LF}

Determining the faint-end slope of the galaxy LF is crucial to constrain the physics governing galaxy formation and evolution at the smallest scales and to understand the relation between stellar content and dark matter halo in dwarf galaxies. The "missing satellite" problem around the MW implies a shallower LF slope ( -1.2; e.g., Koposov et al. 2008) than that predicted for the mass function of dark matter subhalos ( -2.0; e.g., Trentham \& Tully 2002). Several possible explanations (in terms of both observational incompleteness and theoretical modeling) have been put forward in the past decade to address this issue (e.g., Tollerud et al. 2008; Brooks et al. 2013; Hargis et al. 2014; Sawala et al. 2016; Wetzel \& Hopkins 2016; Garrison-Kimmel et al. 2017; Kim et al. 2018): the general consensus is that the incorporation of prescriptions for feedback, star formation efficiency, and reionization into cosmological simulations can help reconcile the observed LF slope with theoretical predictions (see the recent review by Bullock \& Boylan-Kolchin 2017). However, the questions whether or not such models are tuned for the MW LF and whether or not they are capable of reproducing other systems remain.

The faint end of the satellite LF of further systems beyond the Local Group is key to understanding the typical LF slope and its scatter from system to system.

Beyond the Local Group, the measurement of the LF is complicated by two factors: the detection limits for satellites are significantly brighter and quickly fade with distance, and assessing the membership of candidate satellite galaxies with distance/velocity measurements is often prohibitively expensive. Perhaps not surprisingly, contrasting results have been derived for the LF in galaxy cluster and field environments, pointing to a possible dependence on environmental density (see, e.g., Ferrarese et al. 2016, and references therein). Here we focus on a sample of nearby $(<10 \mathrm{Mpc})$ groups of galaxies, for which satellite memberships have been confirmed. With the aim to obtain as fair a comparison as possible in a range of group environments, we choose to perform an area-limited comparison.

We compile the cumulative LFs (CLFs) for the Local Group and for nearby groups of galaxies with satellites confirmed via distance measurements. For the MW, we adopt the updated online 2015 version of the McConnachie (2012) compilation; since the limiting magnitude for known dwarfs around the MW is significantly fainter than for all other groups, we only consider objects with $M_{V}<-5$, thus practically excluding all the recent extremely faint discoveries, e.g., from the DES (except for Eridanus II, which has $M_{V}=-7.1$; see Crnojević et al. 2016b). For M31, we combine the catalogs presented in Martin et al. (2016) and McConnachie et al. (2018). Arguably, M31 and its subgroup provide the environment for which the LF is best constrained to date, in terms of brightness limits, spatial coverage, and detection completeness: satellites as faint as $M_{V} \sim-6$ have been discovered out to a radius of approximately $150 \mathrm{kpc}$ (M31's virial radius is estimated to be $\sim 300 \mathrm{kpc}$ ) over the course of the past decade (see McConnachie 2012, and references therein).

For galaxies beyond the Local Group, our main sources are the Updated Nearby Galaxy Catalog (Karachentsev et al. 2013, from which we only select satellites with positive tidal indexes) and EDD ${ }^{11}$ the Extragalactic Distance Database (Jacobs et al. 2009). For M81, we complement these entries with Table 3 from Chiboucas et al. (2013), who performed a CFHT/ MegaCam wide-field survey of M81 to search for faint

\footnotetext{
${ }^{11}$ http://edd.ifa.hawaii.edu/
} 
satellites, which were then confirmed as group members with HST follow-up imaging (see Chiboucas et al. 2009, 2013, for details). From this sample, we exclude possible tidal dwarfs. The M81 reported magnitudes are in $r$ band, $M_{r}$, which we convert to $M_{V}$ adopting the empirical relation derived from our Magellan data set for Cen A dwarfs $\left(M_{V} \sim M_{r}+0.4\right)$. We also note that, for their faintest M81 satellite d0944+69, Chiboucas et al. (2013) report $M_{I}$ and $M_{r}$ that differ by $\sim 2$ mag; thus, the faintest data point of the M81 CLF is highly uncertain. For M101 we also adopt the Karachentsev et al. (2013) catalog, to which we add the three faint satellites discovered by Dragonfly (and later confirmed via distance measurements; see Merritt et al. 2014; Danieli et al. 2017). Three galaxies close to M101 (NGC 5474, NGC 5477, and UGC 9405) do not have direct TRGB distance measurements but are considered as likely distant group members. The M94 spiral has been recently surveyed by Smercina et al. (2018), who added two faint satellites with TRGB distances to only two other likely distant group members (KK 160 and IC 3687), making this the most poorly populated environment of our sample. Finally, for Cen A we complement the results from this paper with the catalog from Karachentsev et al. (2013): the listed $M_{B}$ magnitudes are transformed into $M_{V}$ by applying the conversion $M_{V}=M_{B}-0.31$. This relation is derived for a subsample of satellites for which $M_{V}$ values are reported in Sharina et al. (2008) and updated by applying the latest distance measurements. We have compiled an updated table of the Cen A satellites with projected distances $<300 \mathrm{kpc}$, including their coordinates, projected distances, and luminosities (Table 6).

The area coverage of the different surveys we consider is not easy to quantify in light of the underlying distribution of satellite galaxies, and as mentioned before, we restrict the derived CLFs by area. The PISCeS survey has been designed to cover $150 \mathrm{kpc}$ in radius around its target galaxies Cen A and Sculptor, offering the advantage of a relatively straightforward comparison to the PAndAS survey of M31. In the bottom panel of Figure 15, we only consider satellites with distances $<150 \mathrm{kpc}$ from the respective host; such distances are necessarily projected, except for the MW, where 3D distances are adopted. In the top panel of Figure 15, we additionally draw the CLF for group members found within the virial radius of each host: the latter is an uncertain quantity, and we assume it to be $\sim 300 \mathrm{kpc}$ given the comparable luminosities of our sample of galaxies (e.g., Klypin et al. 2002). A few caveats are worth mentioning: (i) MW surveys inevitably suffer from incompleteness effects (mainly due to incomplete spatial coverage, especially in the direction of the Galaxy plane), which may underestimate the number of faint satellites by a factor of $\sim 3$ (e.g., Tollerud et al. 2008; Hargis et al. 2014). (ii) For Cen A, a number of candidate satellites have been discovered as unresolved low surface brightness objects in a DECam imaging survey of $\sim 500 \mathrm{deg}^{2}$ around Cen A: Müller et al. (2017) present $\sim 40$ new candidate satellites, of which 13 are located within its virial radius (but none within the PISCeS footprint). These candidates await distance measurements to be confirmed as group members and are not included in the CLF, which could thus be steeper than the one we construct in the magnitude range $-8 \lesssim M_{V} \lesssim-12$. (iii) For M101, a similar search for low surface brightness galaxies has been presented in Bennet et al. (2017); thus, the CLF for this group might also be a lower limit at its faint end $\left(M_{V}>-10\right)$. (iv) A deep search for faint satellites has not been performed beyond the innermost $150 \mathrm{kpc}$ for M94; thus, the CLF within the virial radius for this host is likely a lower limit for $M_{V} \gtrsim-11$.

Although artificial galaxy tests have not been performed yet for PISCeS, we can estimate our dwarf detection incompleteness by considering our discoveries: our limiting absolute magnitude and surface brightness are $M_{V} \sim-8$ and $\sim 26.5 \mathrm{mag} \operatorname{arcsec}^{-2}$, respectively. The major factor impacting our ability to find new dwarfs is the highly varying seeing conditions under which our ground-based survey was performed ( 0."5-1"!0). The PISCeS dwarfs with the lowest central surface brightness values were discovered in fields with seeing in at least one of the bands of 0 ". 65 or better; in terms of absolute magnitude, the same seeing limit allows us to uncover objects with $M_{V} \sim-9$, while the $M_{V} \sim-8$ satellites were found under slightly better seeing conditions $(\sim 0$ !" 6$)$. The dwarf discovered under the worst seeing conditions $(\sim 0$ ". 8$)$ has an absolute magnitude of $M_{V} \sim-10$. Among the PISCeS pointings, $\sim 10 \%, 35 \%$, and $50 \%$ have seeing worse than $0 . " 8$, 0 ! 65 , and 0. , 6 , respectively: we thus assess our completeness to be around $\sim 90 \%, 70 \%$, and $50 \%$ for absolute magnitudes of $M_{V} \sim-10,-9$, and -8 , respectively. The incompleteness limits will additionally depend on the dwarfs' stellar concentration, on their distribution around Cen A, and on spatial coverage (e.g., galaxies not detected because of bright foreground stars; see Chiboucas et al. 2009; Smercina et al. 2018), but these factors will not significantly alter our main conclusions. Our faintest discoveries all have half-light radii in the range of $0.2-0.6$ !: even if we had not resolved them into stars, the compact size would have likely allowed us to identify them visually as unresolved low surface brightness objects (as for our unresolved candidates that turned out to lie in the background; see Section 4.2). The regime we are least sensitive to is the one at low surface brightness and large half-light radii, with an extremely faint unresolved component: the pointings containing Dw1 and Dw3 (our brightest and most diffuse discoveries) had excellent seeing conditions $(<0$ ". 55 in both bands), and PISCeS is thus not suited to uncovering faint $\left(M_{V} \gtrsim-13\right)$ galaxies with such properties, assuming that they exist (see Figure 11). With these numbers in mind, in the magnitude range $-10<M_{V}<-8$ we might be missing 5-10 galaxies: adding those to Cen A's CLF would not alter its slope within the uncertainties. As mentioned earlier, there are 13 additional unconfirmed candidates with galactocentric distances between 150 and $300 \mathrm{kpc}$ from Müller et al. (2017) in the magnitude range $-12<M_{V}<-8$; these were discovered from integrated light, and a fraction of them could be background objects (none of our unresolved candidates were confirmed as a real Cen A satellite; Section 4.2); thus, we do not consider them further. That said, the Müller et al. (2017) study did recover several of the dwarfs originally found by PISCeS (Crnojević et al. 2016a), so there may yet be true Cen A satellites to be confirmed at such large radii.

We fit a Schechter function to each CLF within the virial radius,

$$
N(<M)=\phi_{*} \gamma\left[\alpha+1,10^{0.4\left(M_{*}-M\right)}\right],
$$

and we report the best-fit $\alpha$ values in the caption of Figure 15 ( $\phi_{*}$ and $M_{*}$ are not well constrained, but they do not significantly affect the slope $\alpha$; see also Chiboucas et al. 2013; Park et al. 2017). The slopes are consistent among the different groups, as well as with previous literature results. In 
both galactocentric distance ranges, we observe a large scatter in the CLFs at fixed magnitude, but there is not an obvious link between the CLF faint-end slope and the luminosity of the giant host, which is similar among the considered groups.

\section{Discussion and Conclusions}

We have presented $H S T$ follow-up imaging of seven newly discovered dwarfs in our PISCeS panoramic survey of Cen A; four additional candidate satellites were found not to be group members as evidenced by a lack of resolved populations in the HST images. We also discovered two more dwarfs in our ground-based 2017 Magellan imaging, bringing the total number of new PISCeS Cen A satellites to 11 (also including the two objects presented in Crnojević et al. 2016a for which $H S T$ data were not obtained). Before PISCeS, 13 satellites were known within its estimated virial radius $(\sim 300 \mathrm{kpc})$, of which five were located within $150 \mathrm{kpc}$ of the giant elliptical; our sample thus almost doubles Cen A's satellite population.

The exquisitely deep HST imaging allowed us to derive updated values for the distances, luminosities, structural parameters, and photometric metallicities for the target Cen A dwarfs. With respect to the discovery paper (Crnojević et al. 2014a), Dw1's absolute magnitude and surface brightness are revised to be significantly brighter, placing this dwarf in the ultradiffuse category; Dw3, which is heavily disrupting, can also be considered an ultradiffuse galaxy at the present time. Both Dw1 and Dw3 are found to host metallicity gradients; the tidal tails of Dw3 are more metal-poor than the surrounding populations in Cen A's halo, indicating that satellites of similar luminosity likely did not contribute to the buildup of its outer halo. All the other discoveries from PISCeS are relatively faint and compact objects. The 11 new PISCeS dwarfs are all predominantly old with no signs of recent $(\lesssim 1 \mathrm{Gyr})$ star formation: within $150 \mathrm{kpc}$ of Cen A, the majority of satellites are not currently forming stars, with the exception of ESO 324024, NGC 5237, and KK 196 (all previously known). This will depend on their absolute luminosity (the most luminous satellites have been able to form stars until the present day), as well as on their group infall time (indeed, they are among the most distant dwarfs from Cen A, at least in projection). The PISCeS dwarfs extend the previously known satellite population 2 mag fainter in both absolute magnitude and central surface brightness (Figure 11): among the "classical" dwarfs, only one has $M_{V} \sim-10$, while 9 out of our 11 new discoveries are fainter than this limit, and all of them have central surface brightness values fainter than the previous $\sim 24.5$ mag $\operatorname{arcsec}^{-2}$ limit. No ultrafaint dwarfs were uncovered by PISCeS, but several ultracompact dwarf candidates are being followed up spectroscopically (K. Voggel et al. 2019, in preparation). Finally, the range in half-light radii is extended to both smaller and larger values with respect to the "classical" dwarfs.

Müller et al. (2016) conducted a thorough analysis of both confirmed and candidate dwarfs around Cen A (including our PISCeS discoveries from Crnojević et al. 2016a) to investigate the two possible planes of satellites presented by Tully et al. (2015), concluding that the presence of one single plane is more likely. Recently, Müller et al. (2018) additionally presented evidence for a rotating plane of satellites around Cen A: it will be interesting to collect kinematic data for the PISCeS dwarfs to investigate whether they belong to this whirling plane. We note that there is a visible asymmetry in the spatial distribution of PISCeS dwarfs, with 8 out of 11 dwarfs located to the north of Cen A's minor axis (coincident with its dust lane); curiously, 5 out of our 11 dwarfs are at a galactocentric distance of $\sim 90 \mathrm{kpc}$.

We investigated the CLF of Cen A within 150 and $300 \mathrm{kpc}$ (i.e., the estimated virial radius) and compared it to those of nearby groups with confirmed faint dwarf satellites (the MW, M31, M81, M101, and M94), spanning a range of host galaxy morphologies and environments (from the relatively isolated M94 and M101 to the rich groups of M81 and Cen A). While the derived faint-end slopes for the various groups are consistent within the sample and with previous literature work, the scatter in the CLFs is significant and does not correlate with the host galaxy mass. Recently, Smercina et al. (2018) performed a similar study of the CLFs in the same nearby groups we consider in this work (except Cen A) and showed that simulations cannot reproduce their observed scatter: the solution they put forward is a halo occupation model where the stellar mass-halo mass relation includes an increased scatter, suggestive of a highly stochastic galaxy formation efficiency in dark matter halos. Clearly, this topic deserves further attention from the theoretical standpoint, and dedicated simulations aimed at reproducing the observed CLFs beyond the Local Group are highly desirable.

The authors thank the referee, whose comments helped improve the presentation of our results. D.C. wishes to kindly thank the hospitality of the Carnegie Observatories, where part of this work has been carried out. D.C. warmly thanks M. Rejkuba for useful discussions. Support for this work was partly provided by NASA through grant Nos. HST-GO13856.001 and HST-GO-HST-GO-14259.001-A from the Space Telescope Science Institute, which is operated by AURA, Inc., under NASA contract NAS 5-26555. D.C. acknowledges support from NSF grant AST-1814208; research by D.J.S. is supported by NSF grants AST-1821967, AST1821987, AST-1813708, and AST-1813466; S.P. acknowledges support from NASA grant NNX14AF84G; P.G. and E.T. acknowledge support from NSF grants AST-1010039 and AST-1412504; J.D.S. acknowledges support from NSF grant AST-1412792; J.S. was supported by NSF grant AST-1514763 and a Packard Fellowship; and K.S. acknowledges support from the Natural Sciences and Engineering Research Council of Canada (NSERC). This work was supported in part by National Science Foundation grant No. PHYS-1066293 and the Aspen Center for Physics. This paper uses data products produced by the OIR Telescope Data Center, supported by the Smithsonian Astrophysical Observatory. This research has made use of the NASA/IPAC Extragalactic Database (NED), which is operated by the Jet Propulsion Laboratory, California Institute of Technology, under contract with the National Aeronautics and Space Administration.

Facilities: Magellan Telescopes, Las Campanas Observatory, Chile (Megacam), Hubble Space Telescope.

\section{ORCID iDs}

D. Crnojević (iఠ) https://orcid.org/0000-0002-1763-4128

D. J. Sand (i) https://orcid.org/0000-0003-4102-380X

P. Bennet (1) https://orcid.org/0000-0001-8354-7279

S. Pasetto (1) https://orcid.org/0000-0002-4926-3704

K. Spekkens (1) https://orcid.org/0000-0002-0956-7949

N. Caldwell (i) https://orcid.org/0000-0003-2352-3202

P. Guhathakurta (1) https://orcid.org/0000-0001-8867-4234 
B. McLeod (ib https://orcid.org/0000-0002-2924-2893

J. Strader (1) https://orcid.org/0000-0002-1468-9668

E. Toloba ำ https://orcid.org/0000-0001-6443-5570

\section{References}

Amorisco, N. C., Monachesi, A., Agnello, A., \& White, S. D. M. 2018, MNRAS, 475, 4235

Barnes, D. G., Staveley-Smith, L., de Blok, W. J. G., et al. 2001, MNRAS, 322,486

Beasley, M. A., \& Trujillo, I. 2016, ApJ, 830, 23

Bennet, P., Sand, D. J., Crnojević, D., et al. 2017, ApJ, 850, 109

Bennet, P., Sand, D. J., Zaritsky, D., et al. 2018, ApJL, 866, L11

Boylan-Kolchin, M., Bullock, J. S., \& Kaplinghat, M. 2011, MNRAS, 415, L40

Brooks, A. M., Kuhlen, M., Zolotov, A., \& Hooper, D. 2013, ApJ, 765, 22

Bullock, J. S., \& Boylan-Kolchin, M. 2017, ARA\&A, 55, 343

Carlin, J. L., Sand, D. J., Muñoz, R. R., et al. 2017, AJ, 154, 267

Carlin, J. L., Sand, D. J., Price, P., et al. 2016, ApJL, 828, L5

Carrillo, A., Bell, E. F., Bailin, J., et al. 2017, MNRAS, 465, 5026

Chiboucas, K., Jacobs, B. A., Tully, R. B., \& Karachentsev, I. D. 2013, AJ, 146,126

Chiboucas, K., Karachentsev, I. D., \& Tully, R. B. 2009, AJ, 137, 3009

Crnojević, D., Ferguson, A. M. N., Irwin, M. J., et al. 2013, MNRAS, 432, 832

Crnojević, D., Ferguson, A. M. N., Irwin, M. J., et al. 2014a, MNRAS, 445, 3862

Crnojević, D., Grebel, E. K., \& Koch, A. 2010, A\&A, 516, A85

Crnojević, D., Rejkuba, M., Grebel, E. K., da Costa, G., \& Jerjen, H. 2011, A\&A, 530, A58

Crnojević, D., Sand, D. J., Caldwell, N., et al. 2014b, ApJL, 795, L35

Crnojević, D., Sand, D. J., Spekkens, K., et al. 2016a, ApJ, 823, 19

Crnojević, D., Sand, D. J., Zaritsky, D., et al. 2016b, ApJL, 824, L14

Da Costa, G. S., \& Armandroff, T. E. 1990, AJ, 100, 162

Danieli, S., van Dokkum, P., Merritt, A., et al. 2017, ApJ, 837, 136

Dolphin, A. E. 2002, MNRAS, 332, 91

Dotter, A., Chaboyer, B., Jevremović, D., et al. 2008, ApJS, 178, 89

Drlica-Wagner, A., Bechtol, K., Allam, S., et al. 2016, ApJL, 833, L5

Drlica-Wagner, A., Bechtol, K., Rykoff, E. S., et al. 2015, ApJ, 813, 109

D'Souza, R., \& Bell, E. F. 2018, MNRAS, 474, 5300

Ferrarese, L., Côté, P., Sánchez-Janssen, R., et al. 2016, ApJ, 824, 10

Garrison-Kimmel, S., Wetzel, A., Bullock, J. S., et al. 2017, MNRAS, 471, 1709

Geha, M., Wechsler, R. H., Mao, Y.-Y., et al. 2017, ApJ, 847, 4

Guhathakurta, P., \& Tyson, J. A. 1989, ApJ, 346, 773

Hargis, J. R., Willman, B., \& Peter, A. H. G. 2014, ApJL, 795, L13

Harris, G. L. H. 2010, PASA, 27, 475

Ibata, R. A., Lewis, G. F., Conn, A. R., et al. 2013, Natur, 493, 62

Jacobs, B. A., Rizzi, L., Tully, R. B., et al. 2009, AJ, 138, 332

Janesh, W., Rhode, K. L., Salzer, J. J., et al. 2017, ApJL, 837, L16

Jang, I. S., \& Lee, M. G. 2017, ApJ, 835, 28

Karachentsev, I. D., Makarov, D. I., \& Kaisina, E. I. 2013, AJ, 145, 101

Karachentsev, I. D., Tully, R. B., Dolphin, A., et al. 2007, AJ, 133, 504

Kim, D., \& Jerjen, H. 2015, ApJL, 808, L39

Kim, D., Jerjen, H., Mackey, D., Da Costa, G. S., \& Milone, A. P. 2015, ApJL, 804, L44

Kim, S. Y., Peter, A. H. G., \& Hargis, J. R. 2018, PhRvL, 121, 211302

Kirby, E. N., Cohen, J. G., Guhathakurta, P., et al. 2013, ApJ, 779, 102

Klypin, A., Kravtsov, A. V., Valenzuela, O., \& Prada, F. 1999, ApJ, 522, 82

Klypin, A., Zhao, H., \& Somerville, R. S. 2002, ApJ, 573, 597

Koposov, S., Belokurov, V., Evans, N. W., et al. 2008, ApJ, 686, 279

Koposov, S. E., Belokurov, V., Torrealba, G., \& Evans, N. W. 2015, ApJ, 805,130

Koposov, S. E., Walker, M. G., Belokurov, V., et al. 2018, MNRAS, 479, 5343

Kroupa, P. 2001, MNRAS, 322, 231

Laevens, B. P. M., Martin, N. F., Bernard, E. J., et al. 2015a, ApJ, 813, 44
Laevens, B. P. M., Martin, N. F., Ibata, R. A., et al. 2015b, ApJL, 802, L18

Leaman, R., Venn, K. A., Brooks, A. M., et al. 2013, ApJ, 767, 131

Lee, M. G., Freedman, W. L., \& Madore, B. F. 1993, ApJ, 417, 553

Lianou, S., Grebel, E. K., \& Koch, A. 2011, A\&A, 531, A152

Lim, S., Peng, E. W., Côté, P., et al. 2018, ApJ, 862, 82

Madore, B. F., Mager, V., \& Freedman, W. L. 2009, ApJ, 690, 389

Makarov, D., Makarova, L., Rizzi, L., et al. 2006, AJ, 132, 2729

Martin, N. F., de Jong, J. T. A., \& Rix, H.-W. 2008, ApJ, 684, 1075

Martin, N. F., Ibata, R. A., Lewis, G. F., et al. 2016, ApJ, 833, 167

Martin, N. F., Nidever, D. L., Besla, G., et al. 2015, ApJL, 804, L5

McConnachie, A. W. 2012, AJ, 144, 4

McConnachie, A. W., Ibata, R., Martin, N., et al. 2018, ApJ, 868, 55

McConnachie, A. W., Irwin, M. J., Ibata, R. A., et al. 2009, Natur, 461, 66

McLeod, B., Geary, J., Conroy, M., et al. 2015, PASP, 127, 366

McQuinn, K. B. W., Skillman, E. D., Dolphin, A. E., Berg, D., \& Kennicutt, R. 2016, ApJ, 826, 21

Merritt, A., van Dokkum, P., \& Abraham, R. 2014, ApJL, 787, L37

Mihos, J. C., Durrell, P. R., Ferrarese, L., et al. 2015, ApJL, 809, L21

Moore, B., Ghigna, S., Governato, F., et al. 1999, ApJL, 524, L19

Müller, O., Jerjen, H., \& Binggeli, B. 2017, A\&A, 597, A7

Müller, O., Jerjen, H., Pawlowski, M. S., \& Binggeli, B. 2016, A\&A, 595, A119

Müller, O., Pawlowski, M. S., Jerjen, H., \& Lelli, F. 2018, Sci, 359, 534

Mutlu-Pakdil, B., Sand, D. J., Carlin, J. L., et al. 2018, ApJ, 863, 25

Park, H. S., Moon, D.-S., Zaritsky, D., et al. 2017, ApJ, 848, 19

Pawlowski, M. S., \& Kroupa, P. 2013, MNRAS, 435, 2116

Pawlowski, M. S., Pflamm-Altenburg, J., \& Kroupa, P. 2012, MNRAS, 423, 1109

Peng, C. Y., Ho, L. C., Impey, C. D., \& Rix, H.-W. 2002, AJ, 124, 266

Planck Collaboration, Ade, N., Aghanim, P. A. R., et al. 2016, A\&A, 594, A13

Rejkuba, M., Harris, W. E., Greggio, L., et al. 2014, ApJL, 791, L2

Rejkuba, M., Harris, W. E., Greggio, L., \& Harris, G. L. H. 2011, A\&A, 526, A123

Rizzi, L., Tully, R. B., Makarov, D., et al. 2007, ApJ, 661, 815

Sakai, S., Madore, B. F., Freedman, W. L., et al. 1997, ApJ, 478, 49

Sand, D. J., Crnojević, D., Bennet, P., et al. 2015a, ApJ, 806, 95

Sand, D. J., Crnojević, D., Strader, J., et al. 2014, ApJL, 793, L7

Sand, D. J., Spekkens, K., Crnojević, D., et al. 2015b, ApJL, 812, L13

Sand, D. J., Strader, J., Willman, B., et al. 2012, ApJ, 756, 79

Sandage, A., \& Binggeli, B. 1984, AJ, 89, 919

Sawala, T., Frenk, C. S., Fattahi, A., et al. 2016, MNRAS, 457, 1931

Sharina, M. E., Karachentsev, I. D., Dolphin, A. E., et al. 2008, MNRAS, 384,1544

Sirianni, M., Jee, M. J., Benítez, N., et al. 2005, PASP, 117, 1049

Smercina, A., Bell, E. F., Price, P. A., et al. 2018, ApJ, 863, 152

Smercina, A., Bell, E. F., Slater, C. T., et al. 2017, ApJL, 843, L6

Springel, V., Frenk, C. S., \& White, S. D. M. 2006, Natur, 440, 1137

Stetson, P. B. 1987, PASP, 99, 191

Stetson, P. B. 1994, PASP, 106, 250

Tollerud, E. J., Bullock, J. S., Strigari, L. E., \& Willman, B. 2008, ApJ, 688, 277

Tollerud, E. J., Geha, M. C., Grcevich, J., Putman, M. E., \& Stern, D. 2015, ApJL, 798, L21

Tollerud, E. J., \& Peek, J. E. G. 2018, ApJ, 857, 45

Toloba, E., Sand, D. J., Spekkens, K., et al. 2016, ApJL, 816, L5

Torrealba, G., Belokurov, V., Koposov, S. E., et al. 2018, MNRAS, 475, 5085

Torrealba, G., Koposov, S. E., Belokurov, V., \& Irwin, M. 2016, MNRAS, 459, 2370

Trentham, N., \& Tully, R. B. 2002, MNRAS, 335, 712

Tully, R. B., Libeskind, N. I., Karachentsev, I. D., et al. 2015, ApJL, 802, L25

van Dokkum, P. G., Abraham, R., Merritt, A., et al. 2015, ApJL, 798, L45

Vogelsberger, M., Genel, S., Springel, V., et al. 2014, MNRAS, 444, 1518

Weisz, D. R., Dolphin, A. E., Skillman, E. D., et al. 2014, ApJ, 789, 147

Wetzel, A. R., Hopkins, P. F., Kim, J.-H., et al. 2016, ApJL, 827, L23 